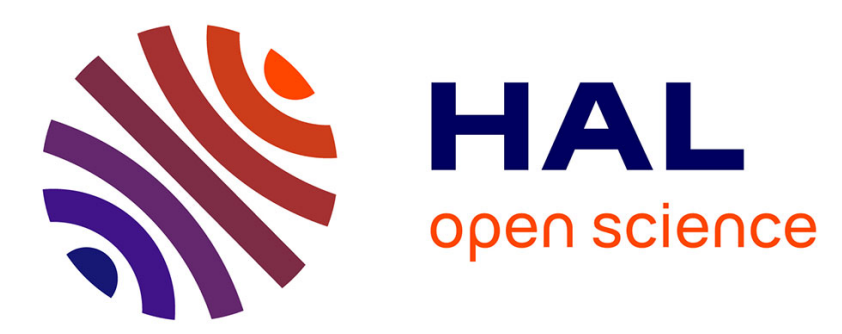

\title{
Induced polarization of volcanic rocks. 4. Large-scale induced polarization imaging
}

\author{
Lutz Gross, A Soueid ahmed, A Revil
}

\section{To cite this version:}

Lutz Gross, A Soueid ahmed, A Revil. Induced polarization of volcanic rocks. 4. Largescale induced polarization imaging. Geophysical Journal International, 2021, 225, pp.950 - 967. 10.1093/gji/ggab018 . hal-03226976

\section{HAL Id: hal-03226976 \\ https://hal.science/hal-03226976}

Submitted on 16 May 2021

HAL is a multi-disciplinary open access archive for the deposit and dissemination of scientific research documents, whether they are published or not. The documents may come from teaching and research institutions in France or abroad, or from public or private research centers.
L'archive ouverte pluridisciplinaire HAL, est destinée au dépôt et à la diffusion de documents scientifiques de niveau recherche, publiés ou non, émanant des établissements d'enseignement et de recherche français ou étrangers, des laboratoires publics ou privés. 


\title{
Induced polarization of volcanic rocks. 4. Large-scale induced polarization imaging
}

\author{
L. Gross ${ }^{\oplus},{ }^{1}$ A. Soueid Ahmed ${ }^{2}$ and A. Revil ${ }^{\oplus 2}$ \\ ${ }^{1}$ The University of Queensland, School of Earth and Environmental Sciences, St Lucia QLD 4067, Brisbane, Australia \\ ${ }^{2}$ Université Grenoble Alpes, USMB, CNRS, EDYTEM, 73000 Chambéry, France.E-mail: andre.revil@univ-smb.fr
}

Accepted 2021 January 12. Received 2021 January 12; in original form 2020 May 5

\begin{abstract}
SUMMAR Y
Thanks to the emergence of new technologies developed with the goal of performing largescale galvanometric induced polarization surveys and thanks a better understanding of the underlying physics of induced polarization, this geophysical method can now be applied in the field of volcanology and geothermal resources assessment. A new approach is developed here for directly inverting the primary and secondary electric fields recorded at a set of independent stations when injecting a primary current. The use of independent stations to measure the primary and secondary electrical fields improves the quality of the data by reducing the capacitive coupling effects inherent to systems based on long cables. It avoids issues associated with using the same electrodes for both current injection and voltage measurements and negative apparent resistivity and chargeability values. With such acquisitions, we can perform true 3-D surveys in areas characterized by complex topography such as volcanoes. The numerical scheme we developed returns as output the electrical conductivity and chargeability fields. The implemented methodology presents several advantages. The first is the use of data types at the stations, for example the electric field intensity, that are independent from the local geometrical station parameters such as electrode spacing and dipole orientation. The second advantage lies in the suitability of the proposed approach to perform large-scale applications since we use a matrix-free approach that does not require the assembly of the Jacobian matrices. The third concerns the possibility of performing the inversion on complex geometries through a consistent use of the finite element method on unstructured meshes in combination with algebraic multigrid preconditioning for the regularization and the solution of the forward and adjoint problems. The computation of 3-D sensitivity maps can also be a real asset in survey design. After validating our approach with a benchmark synthetic case study, we test it on a large-scale induced polarization survey that mimic true field conditions on a volcanic environment with rough topography. Our tests demonstrate the high potential of this electric field approach in volcanology especially for deep $(3 \mathrm{~km})$ imagining of the internal structure of volcanoes, which in turn could improve our understanding of hydrothermal systems and allow the monitoring of active volcanoes and the potential risk of collapse.
\end{abstract}

Key words: Electrical properties; Electrical resistivity tomography (ERT); Tomography.

\section{INTRODUCTION}

The monitoring of volcanic activity using time-lapse geoelectrical methods (i.e. the self-potential method, electrical resistivity tomography induced polarization, magnetometric resistivity and induced polarization) can be important to mitigate the risk associated with volcanic eruptions and flank collapses as underlined in the previous papers of this series (Revil et al. 2017a,b, 2019). These geoelectrical methods can be used to localize hydrothermal systems and monitor geothermal systems during their production (see for instance Ogawa et al. 1999). The self-potential method is a passive geophysical technique whose use is particularly appealing in volcanic environments due its sensitivity to ground water flow. This method has been applied to the characterization of a wide range of thermally active areas (e.g. Lewicki et al. 2003; Revil et al. 2003). Electrical resistivity/conductivity tomography has also been a popular approach for volcano characterization (Revil et al. 2010; Fikos et al. 2012; Lesparre et al. 2014; Soueid Ahmed et al. 2018). Despite its broad use, resistivity/conductivity tomography suffers from limitations related to the ambiguity in interpreting the electrical conductivity distribution, for instance the differentiation between magmatic fluids and altered rocks and between pore space versus 
interfacial contributions (Soueid Ahmed et al. 2018). This hinders the use of electrical resistivity tomography as a stand-alone method to characterize active volcanoes.

The induced polarization method is a galvanic geophysical method that can be seen as a natural extension of the electrical resistivity/conductivity method (Schlumberger 1920). In terms of physical processes, induced polarization refers to the ability of rocks to store reversibly electrical charges under the influence of an applied electrical field (Schlumberger 1920). The geophysical method based on this process provides two properties of interest to characterize volcanic environments namely the normalized chargeability plus a distribution of relaxation times. Normalized chargeability can be used as a proxy of alteration (Revil et al. 2017a,b, 2019).

Despite its broad use in mineral exploration (e.g. Gurin et al. 2013), the use of induced polarization in volcanology still remains extremely sparse. In the previous three papers of this series (Revil et al. 2017a,b, 2019), we have provided a unified petrophysical model for electrical conductivity and normalized chargeability of volcanic rocks. Revil et al. (2018) performed a 3-D resistivity and induced polarization investigations at Krafla volcano, Iceland. Thanks to this method, they managed to image the cation exchange capacity (CEC) and the water content of the volcanic rocks, which is impossible with electrical conductivity tomography alone. Since CEC constitutes a proxy for alteration, these works correspond to the first attempt to image alteration in geothermal fields. Ghorbani et al. (2018) performed induced polarization measurements on 85 core samples from five different volcanoes and provided a complete model able to understand the induced polarization properties of volcanic rocks including the effect of pyrite and magnetite; two semi-conductors that can be present in volcanic environments.

The current technology with long cables allows imaging induced polarization properties to a depth of $\sim 1 \mathrm{~km}$ depending on the conductivity structure of the subsurface, the position of the recording stations, and stacking. A new generation of induced polarization equipment has recently emerged for imaging electrical conductivity and chargeability to greater depths (3-4 km, e.g. Truffert et al. 2017). Such equipment is a wireless system that uses a set of standalone stations laid out at the ground surface (not connected through long cables) to measure the two components of the electric field (the third one, i.e. the component normal to the ground surface is null because the air acts as an insulator). The use of such equipment is appealing for imaging the internal structures of volcanoes and monitoring their shallow activity, especially if we take into account the relative easiness of deploying the measuring stations on terrains characterized by rough topography.

The inversion of induced polarization data has been the subject of relatively intensive research (e.g. Oldenburg \& Li 1994; Li \& Oldenburg 2000; Karaoulis et al. 2011; Fiandaca et al. 2012; Karaoulis et al. 2013; Johnson \& Thomle 2017; Soueid Ahmed et al. 2018). Several commercial and free source codes have been developed for performing electrical resistivity and induced polarization tomography (e.g. Loke 2003; Günther et al. 2006; Johnson et al. 2010; Rücker et al. 2017; Pidlisecky et al. 2007). That said, the development of new approaches for the forward and inverse modeling of induced polarization data is required to be adapted for such equipment for which we can directly use the primary and secondary electric field measurements at a set of independent stations rather than processing potential point measurements over what needs to be considered narrowly spaced electrodes installed at each station. By primary electrical field, we mean the steady state electrical field recorded when the primary current has been injected for a long time. By secondary electrical field, we mean the electrical field decay recorded when the primary current is shut down in a time-domain induced polarization survey.

We present in this paper a novel approach for inverting electrical field measurements to reconstruct the electrical conductivity and chargeability fields of volcanoes. It could also be used to do the tomography of the main relaxation time by inverting a set of apparent chargeability measured for a sequence of time windows. So the present approach can be used to perform full waveform tomography. As the spacing of measuring electrodes of stations are small compared to the extension of the targeted domain fine meshes would be required to locate and separate these electrodes correctly within a potentially rough topography. In order to reduce the required resolution near stations the inversion is based on local electric field data at stations rather than potential differences as conventional approaches do. The resulting focus of inversion sensitivity near stations is reduced through filtering which is applied in form of the inverse of a shifted Laplacian operator applied through the solution of a partial differential equation using the finite element method. This can be read as the application of a second order regularization to the inversion. In addition, we use coordinate system-invariant quantities such as electric field intensity and the projection of the secondary to primary electrical field as data. This avoids potential problems with accuracy when translating between the local, terrain-oriented coordinate systems at each individual station and the global coordinate system of the inversion.

To implement our approach, we added some modifications to the esys-escript package (Schaa et al. 2016). Similar to Codd \& Gross (2017), we use the Algebraic Multi-Grid approach (AMG, Brandt et al. 1985, Brandt 1986, Prokopenko et al. 2014) to solve the forward and adjoint problems. Indeed, the AMG method is versatile and does not require an a-priori knowledge of the geometry of the problem and its spatial discretization. The inverse problem is solved using the limited-memory BFGS (L-BFGS) scheme, which comes with the advantage of not having to explicitly assemble and invert large Jacobian matrices. Adversely to what is predominantly done in the conventional inversion of induced polarization data, we do not minimize for a discretized parameter model in the inverse problem. We minimize for the model parameter as a function of its location in the domain rather than following the common approach formulating and solving an minimization problem in the Euclidean space of model values for a given cell representation (e.g. Gross et al. 2015). This makes the regularization of the inverse problem mesh-independent. We validate our approach on two synthetic case studies. The first corresponds to a simple synthetic example with idealized anomalies. This case is used to validate and showcase the approach. Then, we apply our method to a large-scale induced polarization survey on a synthetic volcano model that reproduces the topography of the Mont St Helens volcano, USA. We simulate a large conduit connected to a deep magmatic chamber and we use only a very limited number of injection points ( 25 in our test). This example includes a fine mesh of millions of cells and serves as a test for showing the robustness of our approach and its high potential for large-scale imaging of the volcanoes internal structures.

\section{THEORETICAL BACKGROUND}

\subsection{Forward modelling}

We consider an induced polarization data collection following the Fullwaver survey design (Truffert et al. 2017) as sketched in Fig. 1. 
This design was created to minimize the number of negative apparent resistivity and chargeability values by measuring the two components of the electrical field tangential to the ground surface. The survey uses a set of current bipoles $A B$ in which the current is injected sequentially between two (current) electrodes A and B. The current electrode A is considered to be fixed while B is mobile and its position are moved sequentially to a set of predefined points in a survey. In addition to the injection system, we consider a set of recording stations in which the two components of the electrical field are recorded at the ground surface. We assume that the electrical field is sampled at a certain sampling frequency (typically one hundred hertz) on a continuous way and both the primary and secondary electrical fields are sampled at each station (Fig. 2). The components of the electrical field are tangential to the ground surface and the third component (normal to the ground surface) is null because of the boundary (insulating) condition at the ground surface. It follows that the two electric potential differences $\Delta V_{i}$ are measured each across two dipoles of electrodes that are orthogonal to each other and are recorded through two independent channels $i=1$ and $i=2$ (Fig. 1). Channel $i=1$ records the potential difference $\Delta V_{1}$ across dipole $i=1$ formed by electrodes at points $P_{2}$ and $P_{3}$ while channel $i=2$ records the potential differences $\Delta V_{2}$ across dipole $i=2$ formed by electrodes at points $P_{2}$ and $P_{1}$. We denote $L_{i}$ the distance between the electrodes of these two dipoles which are forming an $\mathrm{L}$ shape.

For dipole $i(i=1,2)$ and the assumption of a flat Earth surface the apparent resistivity $\rho_{i}^{(a)}$ at a given station is set as

$\rho_{i}^{(a)}=\frac{2 \pi R_{i}}{I} \Delta V_{i} ; \frac{1}{R_{i}}=\frac{1}{r_{i A}}-\frac{1}{r_{i B}}-\frac{1}{r_{O A}}+\frac{1}{r_{O B}}$,

where $I$ (in A) denotes the current injected between the charging electrodes positioned at $\mathrm{A}$ and $\mathrm{B}$. In this formula $r_{O A}$ and $r_{O B}$ (in m) denote the distances of the base electrode $P_{2}$ to the positions A and $\mathrm{B}$ of the charging electrodes, respectively. The corresponding distances of the position of the second dipole electrode - that is $P_{2}$ for dipole $i=1$ and $P_{1}$ for dipole $i=2$ - to the positions A and $\mathrm{B}$ of the charging electrodes are denoted by $r_{i A}$ and $r_{i B}$ (in $\mathrm{m}$ ), respectively. If the orientation of the two dipoles $P_{2}-P_{3}$ and $P_{2}-$ $P_{1}$ are orthogonal, the corresponding axes define a local coordinate system in which the components of the electric field are measured. In practice, the electrodes of each recording station will be oriented in the NS and EW directions. As mentioned above the electrical field is therefore given by

$\mathbf{E}=\left(E_{1}, E_{2}, 0\right)$,

with the following components

$E_{i}=-\frac{\Delta V_{i}}{L_{i}}=-\frac{I}{2 \pi} \frac{\rho_{i}^{(a)}}{L_{i} R_{i}}$.

In order to work with a quantity that is independent of the orientation of the electrodes, we use the electrical field intensity $E$ defined as

$E=|\mathbf{E}|=\sqrt{E_{1}^{2}+E_{2}^{2}}$,

where the Euclidean norm of $\mathbf{p}$ is $|\mathbf{p}|=\sqrt{\mathbf{p}^{T} \mathbf{p}}$ for $T$ denotes the vector transpose. The field intensity can be expressed in terms of the apparent resistivity values $\rho_{1}^{(a)}$ and $\rho_{2}^{(a)}$ :

$E=\frac{I}{2 \pi} \sqrt{\left(\frac{\rho_{1}^{(a)}}{R_{1} L_{1}}\right)^{2}+\left(\frac{\rho_{2}^{(a)}}{R_{2} L_{2}}\right)^{2}}$.
We consider now the secondary field associated with the polarization of the subsurface of a volcano (Fig. 2). Apparent chargeability $\eta_{i}$ along direction $i=1,2$ is defined by

$\eta_{i}=\frac{\Delta \bar{V}_{i}-\Delta V_{i}}{\Delta \bar{V}_{i}}=\frac{\bar{E}_{i}-E_{i}}{\bar{E}_{i}}$,

where $\overline{\mathbf{E}}=\left(\bar{E}_{1}, \bar{E}_{2}, 0\right)$ is the induced polarization (secondary) electric field associated with the chargeability distribution (therefore measured after the primary current between A and B has been shut down). In order to avoid the need to introduce constraints during the inversion process later, we introduce a modified chargeability function:

$\gamma_{i}=\frac{\Delta \bar{V}_{i}-\Delta V_{i}}{\Delta V_{i}}=\frac{\bar{E}_{i}-E_{i}}{E_{i}}=\frac{\eta_{i}}{1-\eta_{i}}$.

Note that $\gamma_{i}$ is non-negative if $\eta_{i}$ is non-negative but in contrast to $\eta_{i}$ its values are not bounded from above. Analogously to the directional modified chargeability we introduce the modified chargeability $\gamma$ as the projection of the relative difference of the electric field difference to the non-induced polarization field $\mathbf{E}$ :

$\gamma=\frac{(\overline{\mathbf{E}}-\mathbf{E})^{T} \mathbf{E}}{E^{2}}=\frac{\hat{\mathbf{E}}^{T} \mathbf{E}}{E^{2}}-1$.

The modified chargeability can be related back with componentwise modified chargeability values $\gamma_{i}$ as

$\gamma=\frac{E_{1}^{2}}{E_{1}^{2}+E_{2}^{2}} \gamma_{1}+\frac{E_{2}^{2}}{E_{1}^{2}+E_{2}^{2}} \gamma_{2}$.

Note that $\gamma_{1}=\gamma_{2}$ leads to $\gamma=\gamma_{1}=\gamma_{2}$. The modified chargeability $\gamma$ can be expressed in terms of the classical chargeability $\eta$ via:

$\eta=\frac{\gamma}{\gamma+1}=\frac{(\overline{\mathbf{E}}-\mathbf{E})^{T} \mathbf{E}}{\overline{\mathbf{E}}^{t} \mathbf{E}}=1-\frac{E^{2}}{\overline{\mathbf{E}}^{t} \mathbf{E}}$

which can be interpreted as the projection of the component-wise classical chargeability definition to the direction of the primary electric field $\mathbf{E}$. A formula for calculating $\eta$ as an average of the component-wise classical chargeabilities $\eta_{i}$ can easily be derived (quantity to be averaged is actually $\left.(1-\eta)^{-1}\right)$. In the inversion, we use the modified chargeability $\gamma$ as the model parameter as it does not require to constrain its values from above.

During a survey, several charging experiments are run. The experiments are indexed by $s$. The forward models provide a prediction of the observations at the stations for an estimated spatial distribution of electric conductivity $\sigma$ and chargeability $\gamma$. A prediction of the electric field intensity $E_{s}$ in charging experiment $s$ is derived from the electric potential $\phi_{s}$ as a solution of the partial differential equation (PDE) of the forward model (for instance Schaa et al. 2016):

$-\nabla^{T} \sigma \nabla \phi_{s}=F_{s}$

where $F_{s}$ represents the source term of a dipole charging experiment $s$. The primary electric field $\mathbf{E}_{s}$ and its magnitude $E_{s}$ is then given as

$\mathbf{E}_{s}=-\nabla \phi_{s} ; E_{s}=\left|\mathbf{E}_{s}\right|$.

Analogously, the secondary electric field $\overline{\mathbf{E}}_{s}$ due to the ground chargeability is given as

$\overline{\mathbf{E}}_{s}=\mathbf{E}_{s}+\hat{\mathbf{E}}_{s}, \hat{\mathbf{E}}_{s}=-\nabla \hat{\phi}_{s}$ and $\bar{E}_{s}=\left|\overline{\mathbf{E}}_{s}\right|$. 


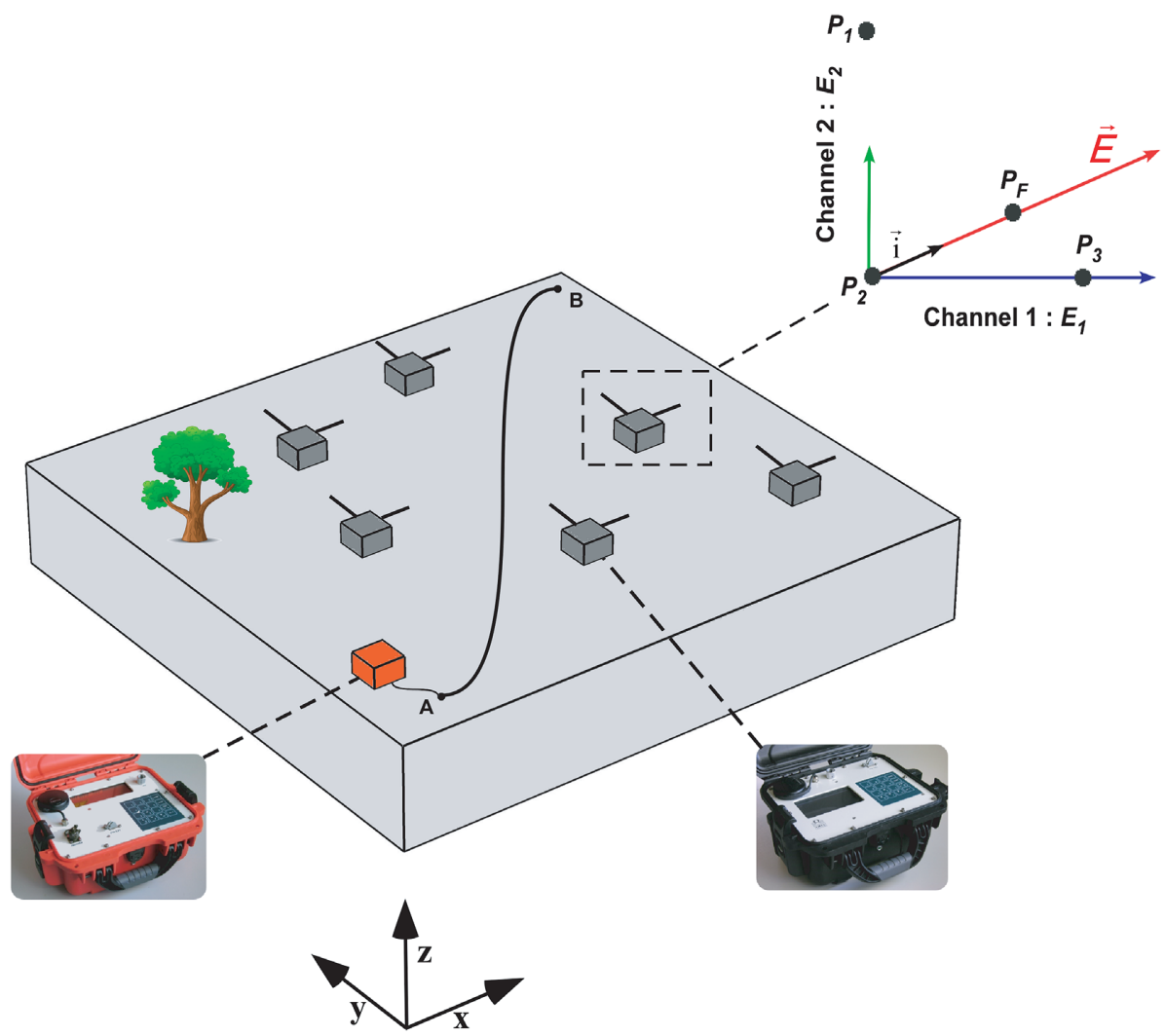

Figure 1. Fullwaver survey design. The Fullwaver system contains two kinds of units: (i) the I-Fullwaver unit (red box) which is used to record time-series of the current injected by the means of a transmitter (ii) the V-Fullwaver (black boxes) which is the recording station that is used to measure the electric field. Several stand-alone stations can be used in a survey. Each station has three electrodes $P_{1}, P_{2}$ and $P_{3}$. The electric field tangential component $E_{1}$ is measured on the first channel between $P_{1}$ and $P_{2}$, while the normal component of the electric field $E_{2}$ is measured on the second channel between $P_{2}$ and $P_{3}$. The quantity $E_{1}$ denotes the component of $\vec{E}$ along the horizontal direction and $E_{2}$ is the component of $\vec{E}$ along the vertical direction. $P_{F}$ denotes a fictitious electrode that can be used to derive the formulation of the apparent resistivity and chargeability. The current is injected between the electrodes A and B, which are connected by a cable. The electrode $A$ remains fixed while the position of $\mathrm{B}$ can be switched for each current injection (modified from Soueid Ahmed et al. 2018).

The secondary potential $\bar{\phi}_{s}$ with $\overline{\mathbf{E}}_{s}=-\nabla \bar{\phi}_{s}$. is given as the solution of the PDE (see Oldenburg \& Li 1994)

$-\nabla^{T} \frac{\sigma}{1+\gamma} \nabla \bar{\phi}_{s}=F_{s}$

where $\gamma$ is the local modified chargeability introduced above. It is noted that for a constant conductivity $\sigma$, the modified chargeability $\gamma$ in eq. (8) and the induced polarization forward model in eq. (14) are consistent in the sense that $\gamma$ as defined in eq. (8) recovers the value for $\gamma$ used in eq. (14).

It is pointed out again that the definition of the electric field intensity $E_{s}$ and the chargeability $\gamma$ are independent of the local coordinate system that is used in the field and hence can directly be compared with the corresponding quantities in the coordinate system used in the inversion without any additional information or coordinate transformations.

Following Schaa et al. (2016) the forward problems corresponding to eqs (11) and (14) are solved in variational form. The formulations are given here as a reference. The variational form of the PDE, eq. (11), is obtained by multiplying the original PDE with an arbitrary but smooth test function $\psi$ and then applying integration by parts with suitable boundary conditions. This results in the condition

$\int_{\Omega} \sigma \nabla^{T} \psi \nabla \phi_{s} \mathrm{~d} x=\int_{\Omega} \psi F_{s} \mathrm{~d} x$,

which needs to be fulfilled for any smooth function $\psi$. The problem is now containing first order derivatives in space only and can easily be solved using the finite element method (FEM) on unstructured meshes (Zienkiewicz et al. 2005). Analogously one derives the variational formulation for the induced polarization problem corresponding to eq. (14) as

$\int_{\Omega} \frac{\sigma}{1+\gamma} \nabla^{T} \psi \nabla \hat{\phi}_{s} \mathrm{~d} x=\int_{\Omega} \frac{\gamma \sigma}{1+\gamma} \nabla^{T} \psi \nabla \phi_{s} \mathrm{~d} x$,

for any smooth function $\psi$. Standard splitting into a primary potential (here $\phi_{s}$ ) and a secondary potential $\left(\right.$ here $\hat{\phi}_{s}$ ) is applied to solve directly for electric field increment $\hat{\mathbf{E}}_{s}=-\nabla \hat{\phi}_{s}$.

\subsection{Inverse modelling}

The objective of the inversion is to recover the distribution of the electric conductivity $\sigma$ and the chargeability $\gamma$ from observations at the stations. Here we follow the approach to first invert for the conductivity $\sigma$ and then in a second step we invert for the chargeability $\gamma$ using the result of $\sigma$ obtained in the first step. In this section, the 

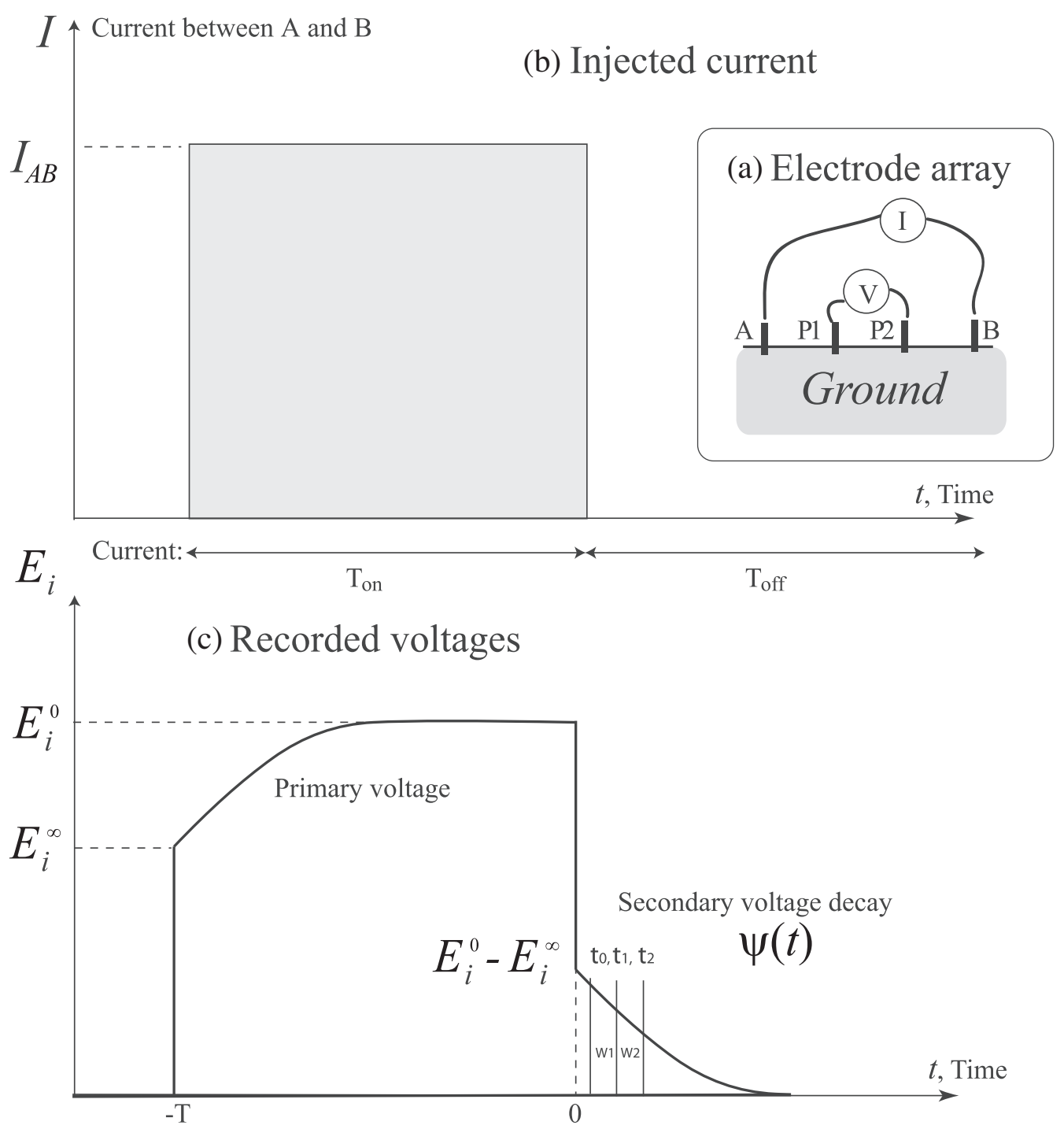

Figure 2. Classical induced polarization sequence in the time domain, with of two distinct chargeability definitions. (a) Electrode array composed of two current electrodes A and B connected to a current generator. Electrodes P1 and P2 are the voltage electrodes connected to a voltmeter characterized by a very high input impedance. (b) Box current characterized by a period $T_{\mathrm{on}}$. The duration between two current injection, $T_{\text {off }}$, should be large enough to allow a full decay of the secondary voltage otherwise the potential would build up in the ground. c. Recorded voltage difference between the electrodes P1 and P2. The buildup of the electrical field during the injection of the current and the decay of the secondary electrical field after shutting down the primary current are symmetrical.

inversion problems are formulated as optimization problems with PDE constraints corresponding to eqs (15) and (16), respectively. The objective function $J_{i}$ ( $i=1$ when inverting for conductivity, $i=2$ when inverting for chargeability) comprises two terms corresponding to the data misfit and regularization terms of the inversion target.

Measurements $D_{s j}$ for the electric field intensity $E_{s}$ are taken at station locations $\mathbf{x}_{j}(j=1, \ldots, M)$ with relative errors $\varepsilon_{E s j}$. Data misfit at station $j$ is evaluated as an average over a small neighbouring region, for instance over a small sphere around location $\mathbf{x}_{j}$. This is introduced because the gradient of a first order FEM solution the field intensity is not continuous across element interfaces and hence has not a unique value at the FEM node coinciding with the station location $\mathbf{x}_{j}$. To mitigate this issue values of the data misfit are locally averaged, for instance over the elements containing the station location $\mathbf{x}_{j}$. This approach also reflects the fact that electric field measurement at a station is derived from potential field measurements over an area and therefore may not give a value at the exact position of the station.

Using a logarithmic scale to emphasize any underestimation of the electric field, this locally averaged misfit is implemented as an integral over the inversion domain $\Omega$ in the form

$M_{1}(\sigma)=\frac{1}{2} \sum_{s} \int_{\Omega} \frac{\chi}{\varepsilon_{E s}^{2}} \cdot \log ^{2}\left(\frac{E_{s}}{D_{s}}\right) \mathrm{d} x$,

where $s$ denotes the injection experiment index, and $E_{s}$ corresponds to the predicted electric field intensity for a given conductivity distribution $\sigma$ obtained by solving the PDE in eq. (11) for the source term representing the injection experiment $s$. The symbol $\log ^{2}$ refers to the square of the natural logarithm function $\log$. The factor $\chi(\mathbf{x})$ is introduced to switch on a contribution $(\chi(\mathbf{x})>0)$ to the average misfit for points $\mathbf{x}$ near a station $\mathbf{x}_{j}$ and suppress any contributions 
$(\chi(\mathbf{x})=0)$ elsewhere in the domain. Corresponding to this setting the function $D_{s}$ of observations is introduced with measured value $D_{s j}$ for locations $\mathbf{x}$ in the neighbourhood of the station located at $\mathbf{x}_{j}$, that is $D_{s}(\mathbf{x})=D_{s j}$, and is set to an arbitrary, positive value otherwise, for instance $D_{s}(\mathbf{x})=1$. Analogously the relative error $\varepsilon_{E s}$ is defined as a function across the inversion domain. Due to construction of the non-negative weighting factor $\chi$ the evaluation of the total misfit $M_{1}(\sigma)$ is collecting contributions from the defect of the electric field intensity $E_{s}$ as a prediction for the observations $D_{s}$ (in a logarithmic scale) as an average over locations nearby a station while potential contributions from other regions are ignored. In this paper we set

$$
\chi(\mathbf{x})= \begin{cases}1 & \text { if }\left|\mathbf{x}-\mathbf{x}_{j}\right| \leq l_{j} \text { for some station at } \mathbf{x}_{j} \\ 0 & \text { otherwise }\end{cases}
$$

With the corresponding definitions for $D_{s}$ and $\varepsilon_{E s}$. The value $l_{j}$ denotes the distance between electrodes $\mathrm{P}_{1}$ and $\mathrm{P}_{3}$ in Fig. 1. The distributed weight function $\chi$ is rescaled such that $\int_{\Omega} \chi \mathrm{d} x=1$. Similarly, the misfit for the apparent chargeability $\gamma_{s}$ is defined as

$$
M_{2}(\gamma)=\frac{1}{2} \sum_{s} \int_{\Omega} \frac{\chi}{\varepsilon_{\gamma s}^{2}} \cdot\left(\frac{d_{s}-\gamma_{s}}{d_{s}}\right)^{2} \mathrm{~d} x
$$

with measured modified chargeability $d_{s}$ and relative measurement error $\varepsilon_{\gamma s}$ for charging experiment $s$. The predicted modified chargeability $\gamma_{s}$ is the solution of the PDE, see eq. (14), for a given realization of the chargeability distribution $\gamma$.

The objective of the inversion is to recover the conductivity $\sigma$ and then chargeability $\gamma$ that minimize the misfit functions corresponding to eqs (17) and (19), respectively. In order to guarantee positive values for both unknown quantities the inversion targets the logarithms by introducing the new variables $p_{1}$ and $p_{2}$ in the following form

$p_{1}=\log \frac{\sigma}{\sigma_{0}} ; p_{2}=\log \frac{\gamma}{\gamma_{0}}$,

where $\sigma_{0}$ and $\gamma_{0}$ are known reference conductivity and chargeability, respectively. Their values could be spatially variable, for instance when other prior geological information are available. The values of the modified chargeability are positive and not bounded from above which frees $p_{2}$ from any constraint that need to be applied during the inversion process. The resulting simplification for the inversion process is the reason why the modified chargeability $\gamma$ is preferred over the standard chargeability $\eta$ which is expected to be bounded between zero and one.

In order to manage the ill-posedness of the inversion problem a regularization term is added to respective misfit functions. Here a second order derivative regularization is applied as

$R\left(m_{i}\right)=\frac{1}{2} \int_{\Omega}\left|m_{i}\right|^{2} \mathrm{~d} x$,

where the property functions $m_{i}$ are given (essentially) as the second order derivative of $p_{i}$ :

$m_{i}=p_{i}-\alpha \Delta p_{i}$ for $i=1,2$.

The factor $\alpha \geq 0$ has to be non-negative. The impact of the choice of this factor on the inversion result is discussed in more details in Section 2.3 and it should be kept in mind that other regularization techniques could be used such as for instance imageguided inversion (Zhou et al. 2014). The second derivative of the functions $p_{1}$ and $p_{2}$ define the property functions $m_{1}$ and $m_{2}$ which are the unknowns of the inversion. The parameter $p_{i}$ is a smoothed version of the property function $m_{i}$ where $\sqrt{\alpha}$ sets the length scale
Table 1. Synthetic test case: Inversion iteration is terminated when relative change in the solution is less than $10^{-4}$. The coefficient of determination $R^{2}$ measures the quality of the logarithm of the recovered anomaly versus the logarithm of the assumed anomaly within the target region (with value one indicating a good and value zero indicating a bad recovery).

\begin{tabular}{lcc}
\hline Parameter & Conductivity & Chargeability \\
\hline Initial misfit & $3.40 \mathrm{e}-02$ & 6.61 \\
Final misfit & $5.69 \mathrm{e}-05$ & $5.36 \mathrm{e}-04$ \\
$R^{2}$ & 0.683 & 0.537 \\
Iterations & 424 & 302 \\
\hline
\end{tabular}

Table 2. Case study 2 statistics. Inversion iteration ends when relative change in the solution is less than $10^{-4}$. The coefficient of determination $R^{2}$ measures the quality of the recovered anomaly, see Table 1 .

\begin{tabular}{lcc}
\hline Parameter & Conductivity & Chargeability \\
\hline Initial misfit & 1.41 & $2.97 \mathrm{e}-01$ \\
Final misfit & $7.30 \mathrm{e}-04$ & $1.40 \mathrm{e}-03$ \\
$R^{2}$ & 0.746 & 0.552 \\
Iterations & 180 & 301 \\
\hline
\end{tabular}

of smoothing. In general, its value can be different if inverting for conductivity or chargeability but we found that in most application cases we have investigated it is appropriate to choose the same value for both inversions. Following the analogous steps to translate the strong formulation of the forward problem (11) to its equivalent variational form (15) the smoothing problem as given in eq. (22) is transformed in variational form as

$\int_{\Omega}\left(q p_{i}+\alpha \nabla^{T} q \nabla p_{i}\right) \mathrm{d} x=\int_{\Omega} q m_{i} \mathrm{~d} x$

for any smooth function $q$ (Mondal et al. 2020). For a given property function $m_{i}$ eq. (23) needs to be solved to obtain $p_{i}$ from which conductivity $\sigma$ or chargeability $\gamma$ are calculated via eq. (20).

The task of the inversion is to find the property function that minimizes the cost function

$J_{i}\left(m_{i}\right)=M_{i}\left(p_{i}\right)+\mu_{i} R\left(m_{i}\right)$,

where the regularization term $R(m)$ as defined in eq. (21) leads to minimization of the (essential) second derivative of the logarithm of conductivity and chargeability. The factor $\mu_{i}$ is a positive factor to weighting the regularization over the misfit. In general it is not expected that the values of the regularization factors for electric conductivity $\mu_{1}$ and chargeability $\mu_{2}$ are chosen to be identical. In some cases the same value may be appropriate namely when both quantities are described by a similar spatial distribution. This is the case in the benchmark problems presented later and could be applied for field data as well.

The inverse problem is solved in two steps: First, the cost function $J_{1}$ in eq. (24) is minimized over $m_{1}$ where the electrical conductivity distribution $\sigma$ is constructed by applying the parametrization corresponding to eq. (20) to $p_{1}$, which is obtained as solution of the PDE corresponding to eq. (22). To obtain the misfit $M_{1}$, the forward model needs to be solved for each charging experiment $s$. After the conductivity distribution $\sigma$ minimizing $M_{1}$ has been found, the cost function $J_{2}$ corresponding to eq. (24) is minimized over $m_{2}$ keeping electrical conductivity $\sigma$ constant. Analogously to the construction of the electrical conductivity field $\sigma$, the modified chargeability distribution $\gamma$ is obtained by solving the PDE corresponding to eq. (14) before applying the parametrization in eq. (20). Note that the PDE corresponding to eq. (14) needs to be solved for each charging experiment $s$. 


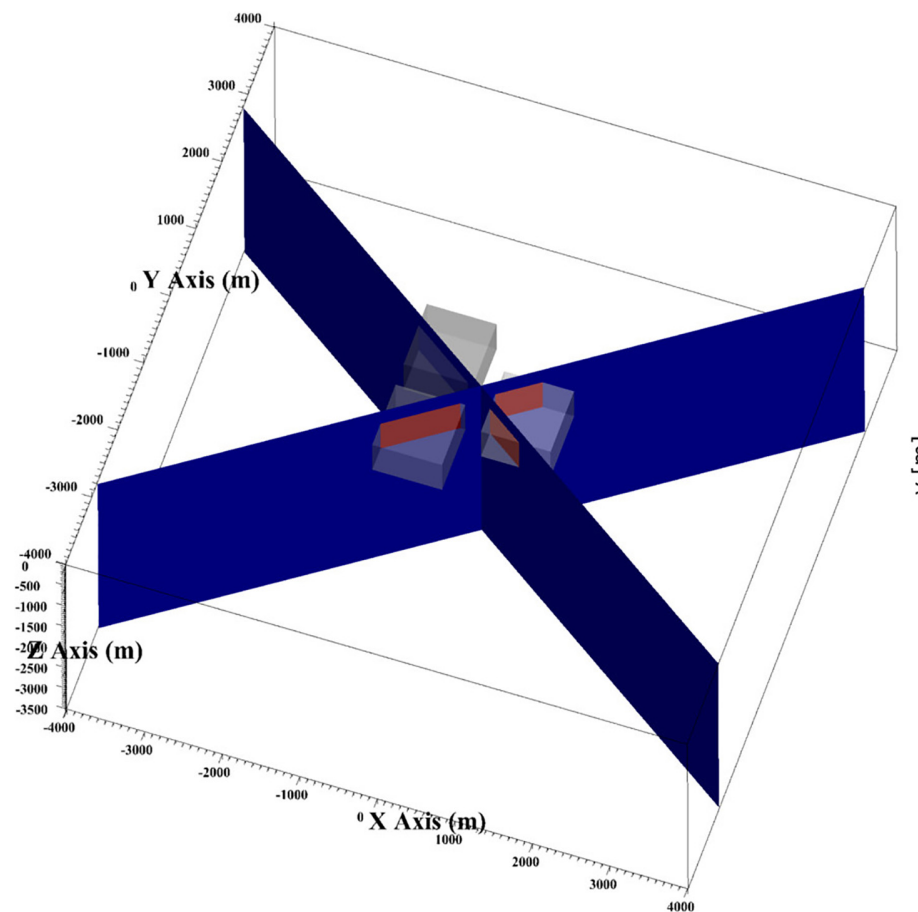

(a) Domain set-up

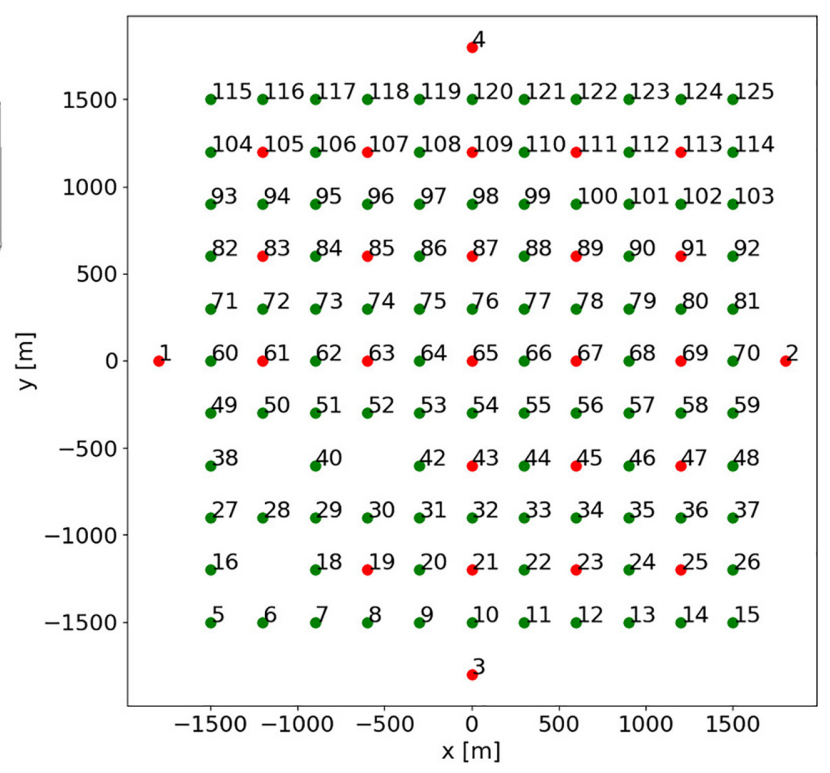

(b) Survey design

Figure 3. Synthetic test case. The domain of interest covers a volume of $4 \times 4 \times 4 \mathrm{~km}^{3}$ with three anomalies of size $900 \times 900 \times 600 \mathrm{~m}^{3}$ at a depth $300 \mathrm{~m}$ embedded in a homogeneous background with conductivity $0.001 \mathrm{Sm}^{-1}$ and chargeability 0.01 . Electric conductivity and chargeability $(\sigma, \gamma)$ in the anomalies are chosen as $\left(0.01 \mathrm{~S} \mathrm{~m}^{-1}, 0.01\right),\left(0.01 \mathrm{~S} \mathrm{~m}^{-1}, 0.20\right)$ and $\left(0.001 \mathrm{~S} \mathrm{~m}^{-1}, 0.20\right)$, respectively. We use 96 recording stations (marked yellow in b) and 26 injection electrodes (marked blue in b) for 40 charging experiments, which correspond to a realistic field survey. The vertical plan in (a) which are positioned at angles $35^{\circ}$ and $-35^{\circ}$ to the $x$-axis are used for presenting 2-D slices of the inversion results.

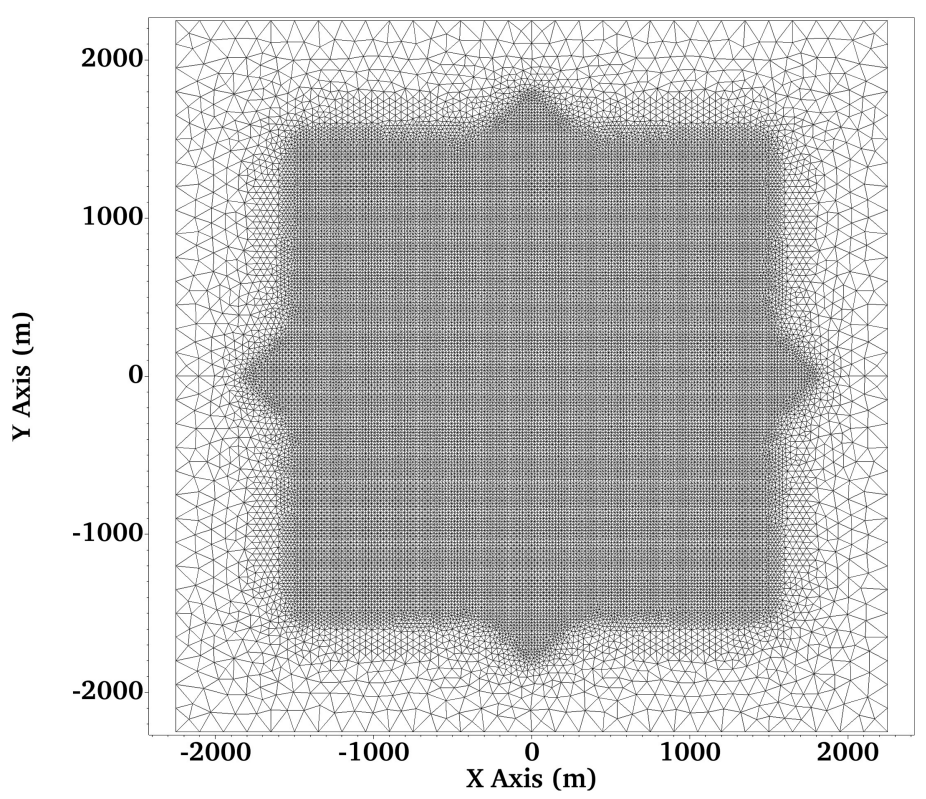

Figure 4. Synthetic test case: top view of the finite-element mesh with 748350 tetrahedral elements. Element size near the $11 \times 11$ grid of electrodes is $35 \mathrm{~m}$. The core regions which is covering the region of interest including the anomalies is meshed with an element size of $150 \mathrm{~m}$ while a mesh size of $600 \mathrm{~m}$ is used in the outer region.

The inversion problems are solved by finding the property functions $m_{i}(i=1,2)$ for which the gradient of the cost function is zero, that is

$$
\left\langle\nabla J_{i}\left(m_{i}\right), \delta m_{i}\right\rangle=0
$$

for all admissible increments $\delta m_{i}$. The term 'admissible increment' refers to an increment $\delta m_{i}$ for which with $m_{i}$ also $m_{i}+\omega \cdot \delta m_{i}$ is still in the space of possible inversion solution for any sufficiently small but positive number $\omega$. The problem (25) is solved using the Broyden-Fletcher-Goldfarb-Shanno (BFGS) iteration scheme 


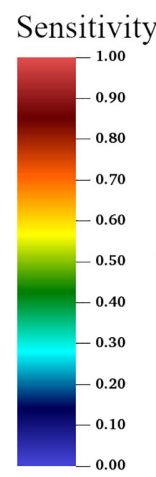

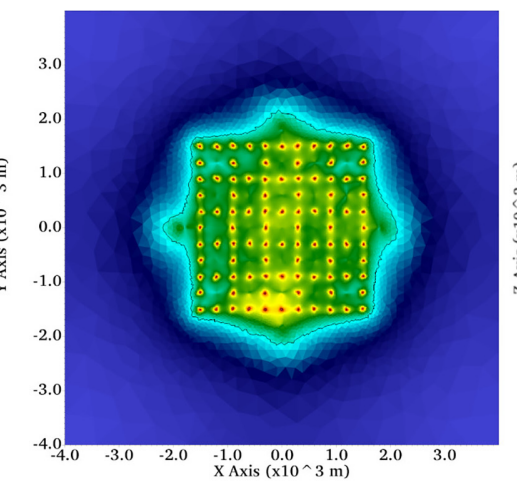

(a) $\alpha=10000$; top view at $z=0$

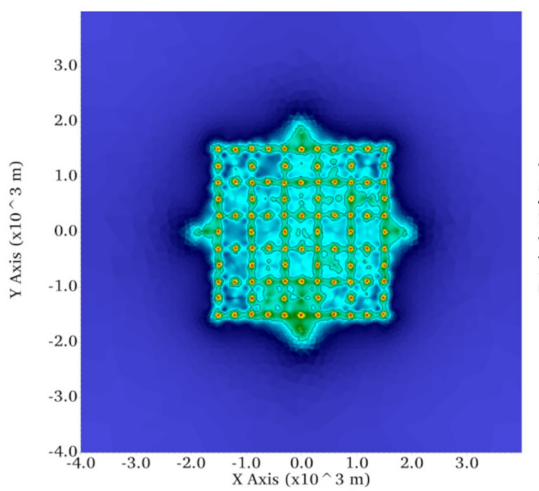

(c) $\alpha=90000$; top view at $z=0$

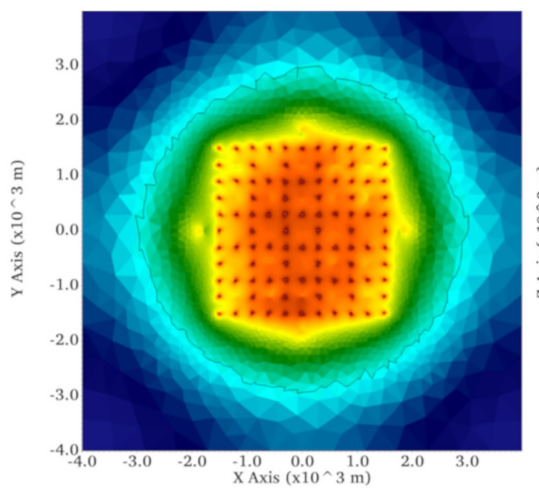

(e) $\alpha=810000$; top view at $z=0$

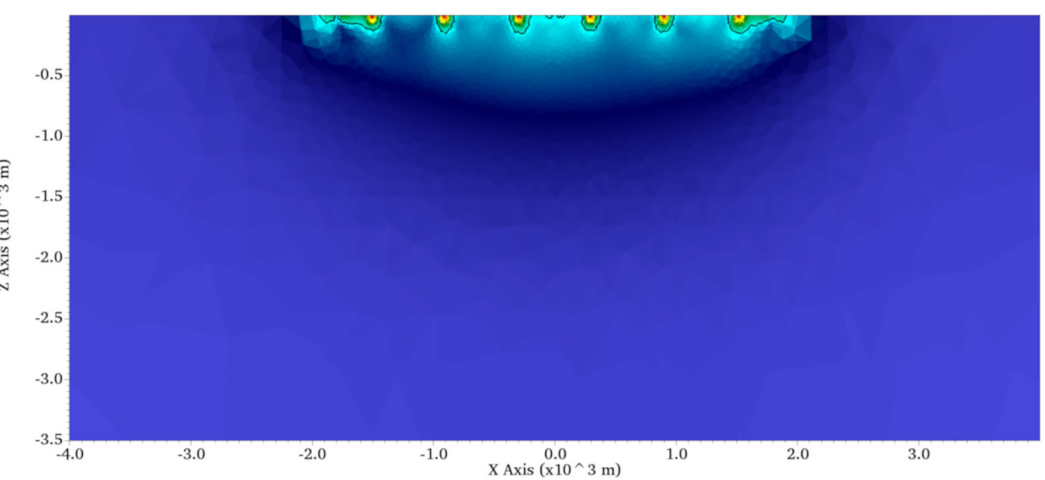

(b) $\alpha=10000$; transect at $y=0$

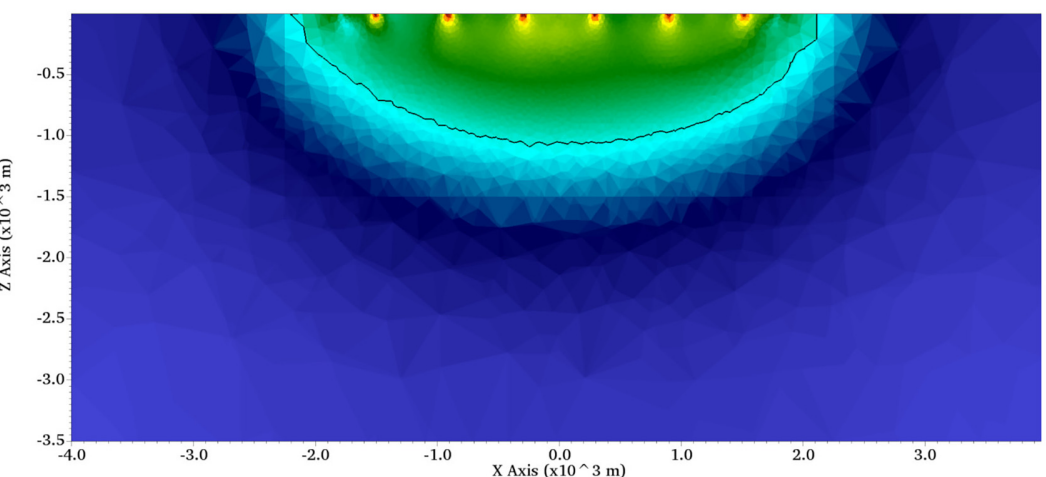

(d) $\alpha=90000$; transect at $y=0$

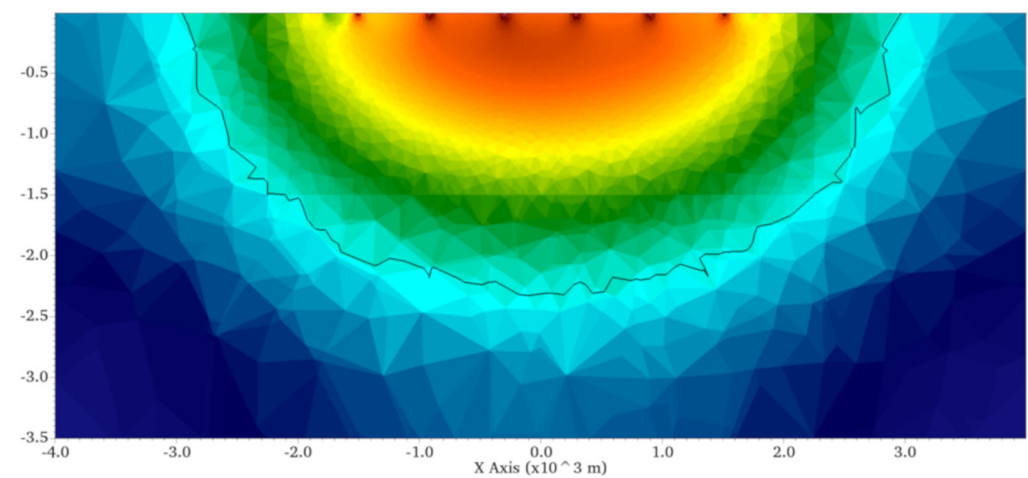

(f) $\alpha=810000$; transect at $y=0$

Figure 5. Sensitivity maps for the synthetic test case (vertical transect at $y=0$ and top view at $z=0$ ) for various values of the length scale parameter $\alpha$ in square meters. Colour shows the sensitivity averaged across all charging experiments scaled by the maximum value using a linear scale. Regions with large sensitivity are shown in red. Low sensitivity regions are coloured in blue. The black line marks the contour for a sensitivity value of 0.3 .

in combination with the finite element method (FEM) following the approach by Gross et al. (2015) and Codd \& Gross (2017). The key difference to the conventional BFGS scheme, which is based on the standard Euclidean norm, is the use of an integral based norm to acknowledge the fact that for an unstructured FEM mesh the locations of FEM nodes used to represent the inversion unknowns could be unevenly distributed. The PDEs for the forward problems, the smoothing step and the BFGS pre-conditioner are all discretized using the same FEM mesh and solved with algebraic multigrid (Codd \& Gross 2017). A central step in the BFGS iteration is the calculation of the cost function gradient $\nabla J_{i}$ for a given $m_{i}$, which needs to be constructed explicitly in each iteration step of the BFGS scheme. Following the idea of a Quasi-Newton scheme, the BFGS constructs approximations of the inverse of the Hessian as a symmetric product of computationally sparse rank one updates which is improved in the course of the iteration using the current cost function gradient. This avoids the explicit construction and inversion of the Hessian that allows applying the BFGS to largescale inversion problems as solved in this paper.

As shown in Gross et al. (2015), the evaluation of the gradient in the given direction $\delta m_{i}$ can be written as

$$
\left\langle\nabla J_{i}\left(m_{i}\right), \delta m_{i}\right\rangle=\int_{\Omega}\left(\mathbf{X}_{i}^{T} \nabla \delta m_{i}+Y_{i} \delta m_{i}\right) \mathrm{d} x
$$

for all admissible increments $\delta m_{i}$ with a suitable vector valued coefficients $\mathbf{X}_{i}$ and a scalar coefficient $Y_{i}$ both depending on $m_{i}$. In the following, we will derive an explicit form for $\nabla J_{i}$. 


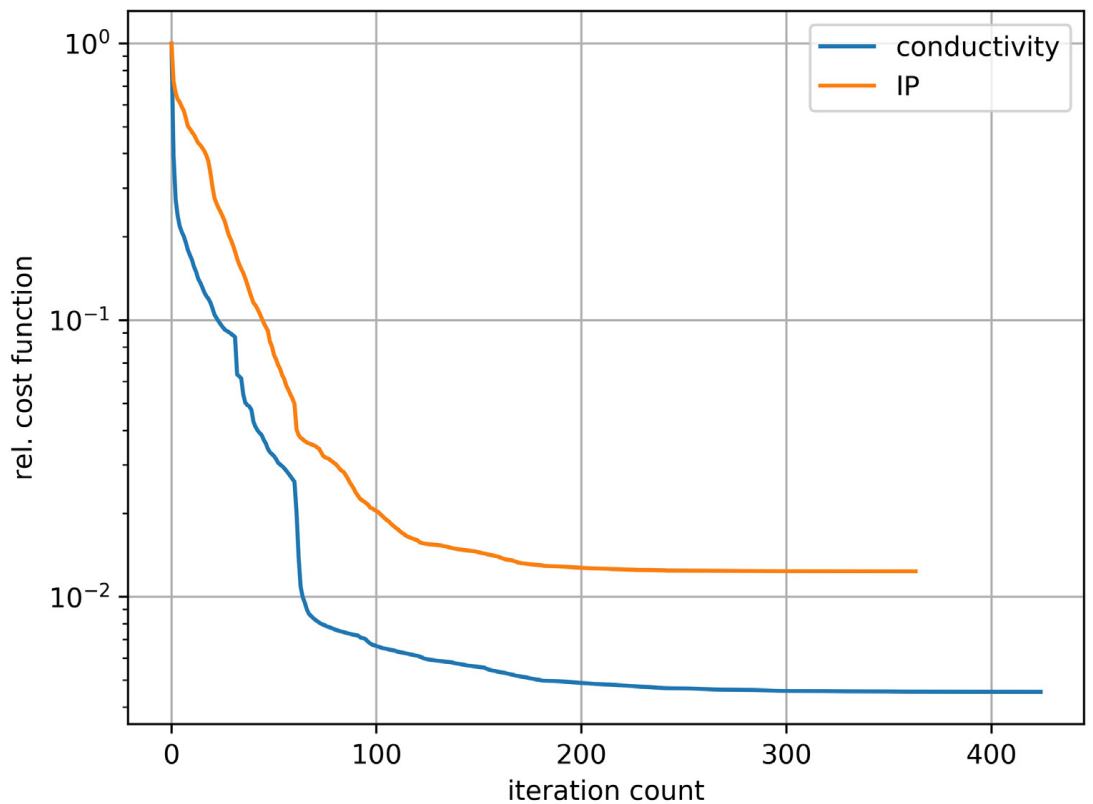

Figure 6. Synthetic test case: convergence history plotting the BFGS iteration count versus the value of the cost function relative to its initial value for conductivity (blue) and induced polarization (IP) chargeability (amber). Note that by construction, the cost function is monotonously decreasing.

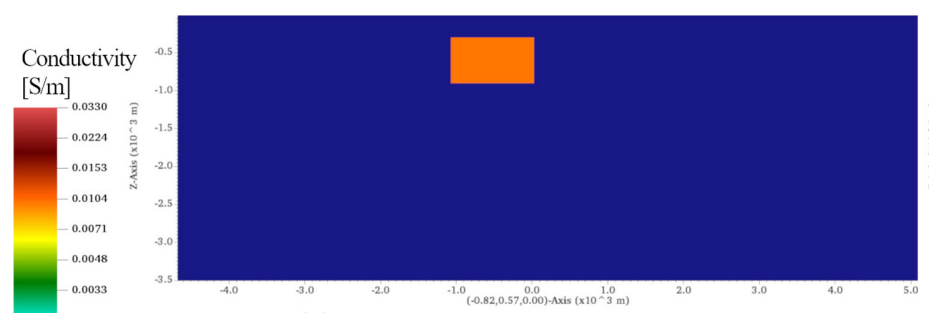

(a) True conductivity at $35 \mathrm{deg}$

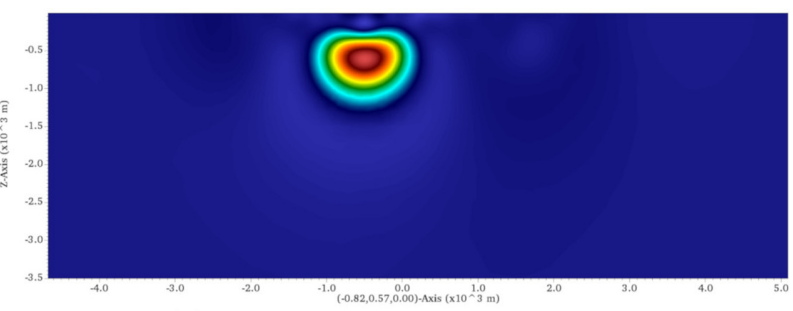

(c) Inverted conductivity at $35 \mathrm{deg}$

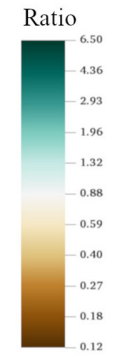

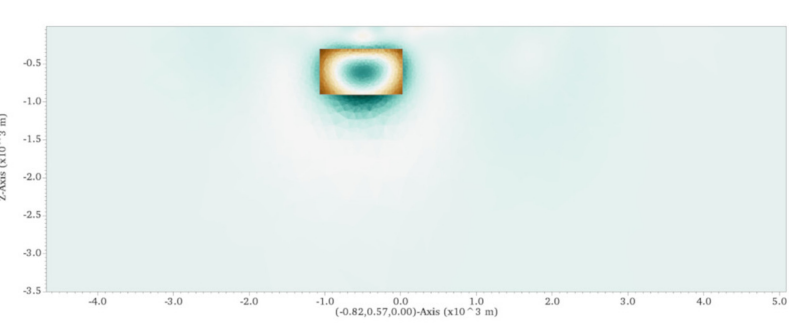

(e) Ratio of true and inverted conductivity at $35 \mathrm{deg}$

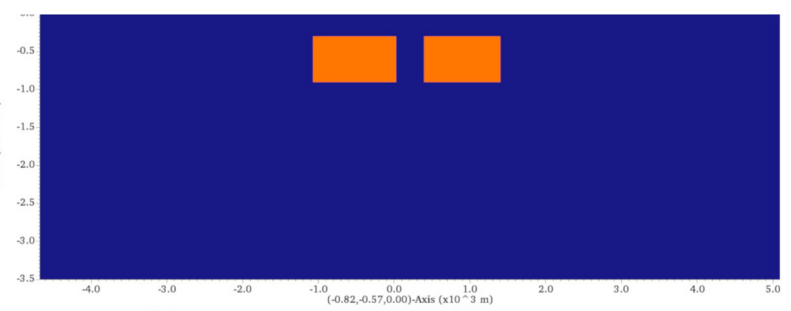

(b) True conductivity at $-35 \mathrm{deg}$

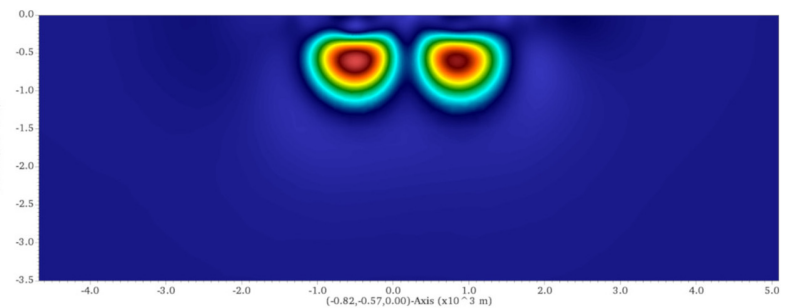

(d) Inverted conductivity at $-35 \mathrm{deg}$

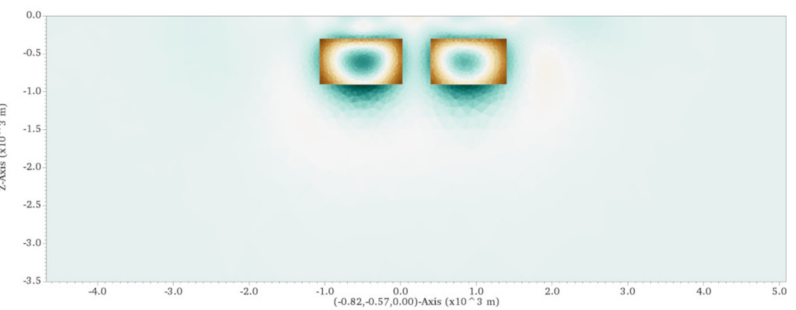

(f) Ratio of true and inverted conductivity at $-35 \mathrm{deg}$

Figure 7. Synthetic test case: Inversion of electrical conductivity $\sigma$. Images show the true conductivity $\sigma_{\text {true }}$, the inverted conductivity $\sigma$ and the logarithm of the ratio $\sigma / \sigma_{\text {true }}$ as a measure of the local error of recovery. The values are shown at the planes at an angle of $35^{\circ}$ and $-35^{\circ}$ to the $x$-axis, see also Fig. $3($ a).

To construct the gradient $\nabla M_{1}$, we first construct the adjoint defect $\phi_{s}^{*}$, which is the solution of the following adjoint variational problem

$$
\int_{\Omega} \sigma \nabla^{T} \phi_{s}^{*} \nabla \psi \mathrm{d} x=\int_{\Omega} \frac{\chi}{\left(\varepsilon_{E s} E_{s}\right)^{2}} \log \left(\frac{E_{s}}{D_{s}}\right) \mathbf{E}_{\mathbf{s}}{ }^{T} \nabla \psi \mathrm{d} x
$$

for any smooth function $\psi$. $\mathbf{E}_{\mathbf{s}}$ is the electric field due to the conductivity distribution $\sigma$. This problem needs to be solved for each charging experiment $s$. As a side note, the adjoint defect $\phi_{s}^{*}$ is identical to zero if the electric field matches the data at all stations. In contrast to solving adjoint problems for each individual station 


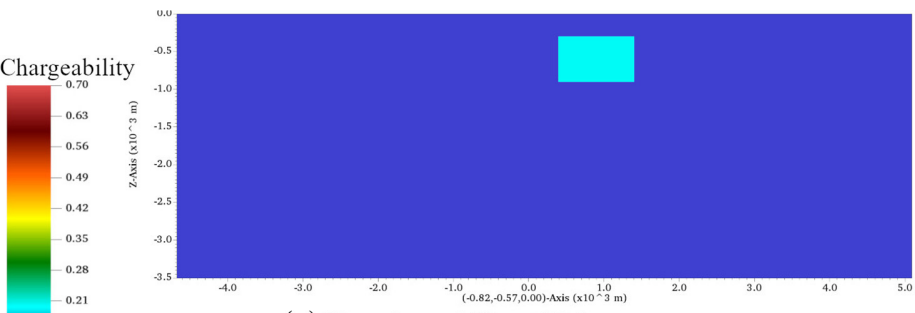

(a) True chargeability at $35 \mathrm{deg}$

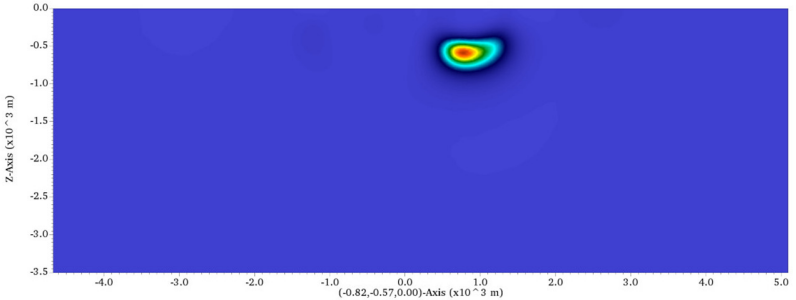

(c) Inverted chargeability at $35 \mathrm{deg}$

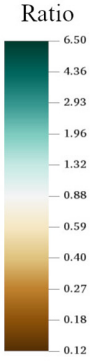

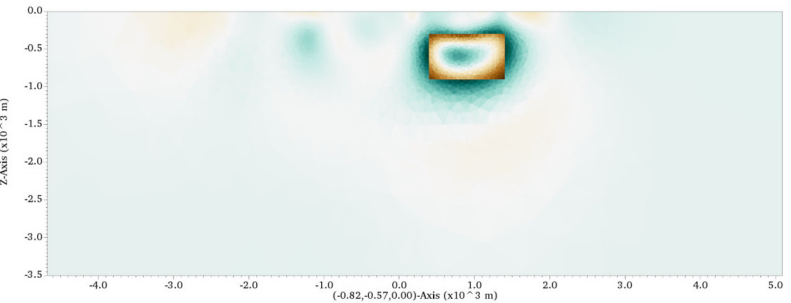

(e) Ratio of true and inverted chargeability at $35 \mathrm{deg}$

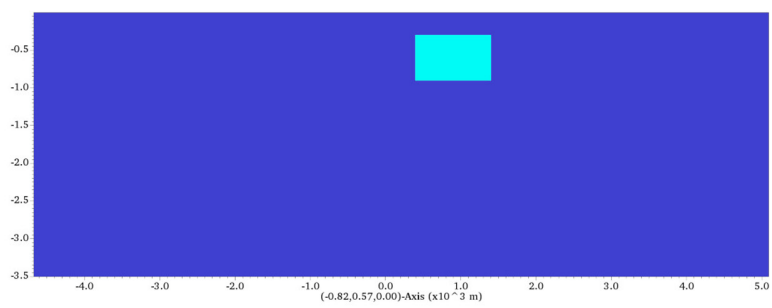

(b) True chargeability at $-35 \mathrm{deg}$

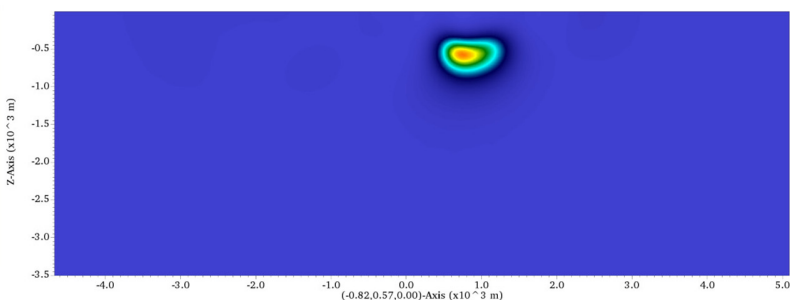

(d) Inverted chargeability at $-35 \mathrm{deg}$

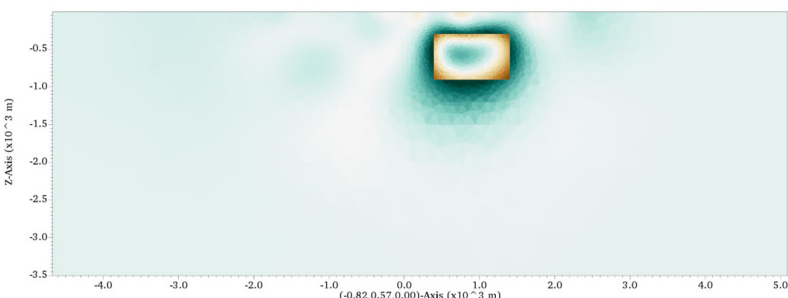

(f) Ratio of true and inverted chargeability at $-35 \mathrm{deg}$

Figure 8. Synthetic test case: Inversion of chargeability $\eta$. Images show the true chargeability $\gamma_{\text {true }}$, the inverted chargeability $\eta$ and the logarithm of the ratio $\eta_{\text {true }} \eta$ as a measure of the local error of recovery. The values are shown at the planes at an angle of $35^{\circ}$ and $-35^{\circ}$ to the $x$-axis, see also Fig. 3 (a).

when assembling the Jacobian matrix the approach taken here combines individual adjoint problem solves to a single problem solve (27) making computational costs independent from the number of stations. To consider the smoothing step (22) an additional variational problem is solved for the adjoint smoothed defect $p_{1}^{*}$, which is given as the solution of the adjoint variational problem

$$
\int_{\Omega}\left(p_{1}^{*} q+\alpha \nabla^{T} p_{1}^{*} \nabla q\right) \mathrm{d} x=\int_{\Omega} q \sigma^{\prime} \sum_{s} \nabla^{T} \phi_{s}^{*} \nabla \phi_{s} \mathrm{~d} x
$$

for any smooth function $q$ where $\sigma^{\prime}$ denotes the derivative of the parametrization of $\sigma$ with respect to $p_{1}$ (see eq. 20). For the construction of $p_{1}^{*}$ only a single variational problem that combines the defects of all charging experiments $s$ needs to solved. The gradient of $M_{1}$ for any variation $\delta m_{1}$ is then given

$$
\left\langle\nabla M_{1}, \delta m_{1}\right\rangle=\int_{\Omega} p_{1}^{*} \delta m_{1} \mathrm{~d} x
$$

The calculation of the gradient for the the misfit of the modified chargeability follows the same idea. First we construct the adjoint defect $\hat{\phi}_{s}^{*}$ by solving the following adjoint problem

$$
\int_{\Omega} \sigma \nabla^{T} \hat{\phi}_{s}^{*} \nabla \psi \mathrm{d} x=\int_{\Omega} \frac{\chi}{\left(\varepsilon_{\gamma s} d_{s} E_{s}\right)^{2}}\left(d_{s}-\gamma_{s}\right) \mathbf{E}_{s}^{T} \nabla \psi \mathrm{d} x
$$

for any smooth function $\psi . \mathbf{E}_{\mathbf{s}}$ is the electric field due to the conductivity distribution $\sigma$ which is fixed when inverting for chargeability. The value $\gamma_{s}$ is the predicted modified chargeability of experiment $\mathrm{s}$ due to the assumed chargeability $\gamma$. An adjoint smoothed defect $p_{2}^{*}$ is introduced by solving the adjoint variational problem

$$
\begin{aligned}
\int_{\Omega}\left(p_{2}^{*} q+\alpha \nabla^{T} p_{2}^{*} \nabla q\right) \mathrm{d} x= & \int_{\Omega} q \frac{\gamma^{\prime} \sigma}{(1+\gamma)^{2}} \\
& \times \sum_{s} \nabla^{T} \hat{\phi}_{s}^{*} \nabla \phi_{s} \mathrm{~d} x,
\end{aligned}
$$

for any smooth function $q$. The value $\gamma^{\prime}$ is the derivative of the parametrization of $\gamma$ with respect to $p_{2}$, see eq. (20). Again while the variational problem corresponding to eq. (30) needs to be solved for all charging experiments $s$, the problem eq. (31) set for the smoothed joint defect $p_{2}^{*}$ needs to be only solved once per iteration step. Analogously to the misfit electric field intensity, the gradient of the misfit $M_{2}$ is given by

$$
\left\langle\nabla M_{2}, \delta m_{2}\right\rangle=\int_{\Omega} p_{2}^{*} \delta m_{2} \mathrm{~d} x .
$$

For the regularization part, $\nabla R$ can easily be obtained as

$$
\left\langle\nabla R\left(m_{i}\right), \delta m_{i}\right\rangle=\int_{\Omega} m_{i} \delta m_{i} \mathrm{~d} x, i=1,2 .
$$

In summary the gradient of the cost function $J_{i}$ is defined by eq. (26) with $Y_{i}=\mu_{i} m_{i}+p_{i}^{*}$ and $\mathbf{X}_{i}=0$.

\subsection{Sensitivity}

A quantity of interest whose evaluation is important is the sensitivity. It can be used to design the optimal locations of the injections and of measurement stations. As sensitivity we define to what extend a change to the inversion target (namely the property function $m_{i}$ ) 


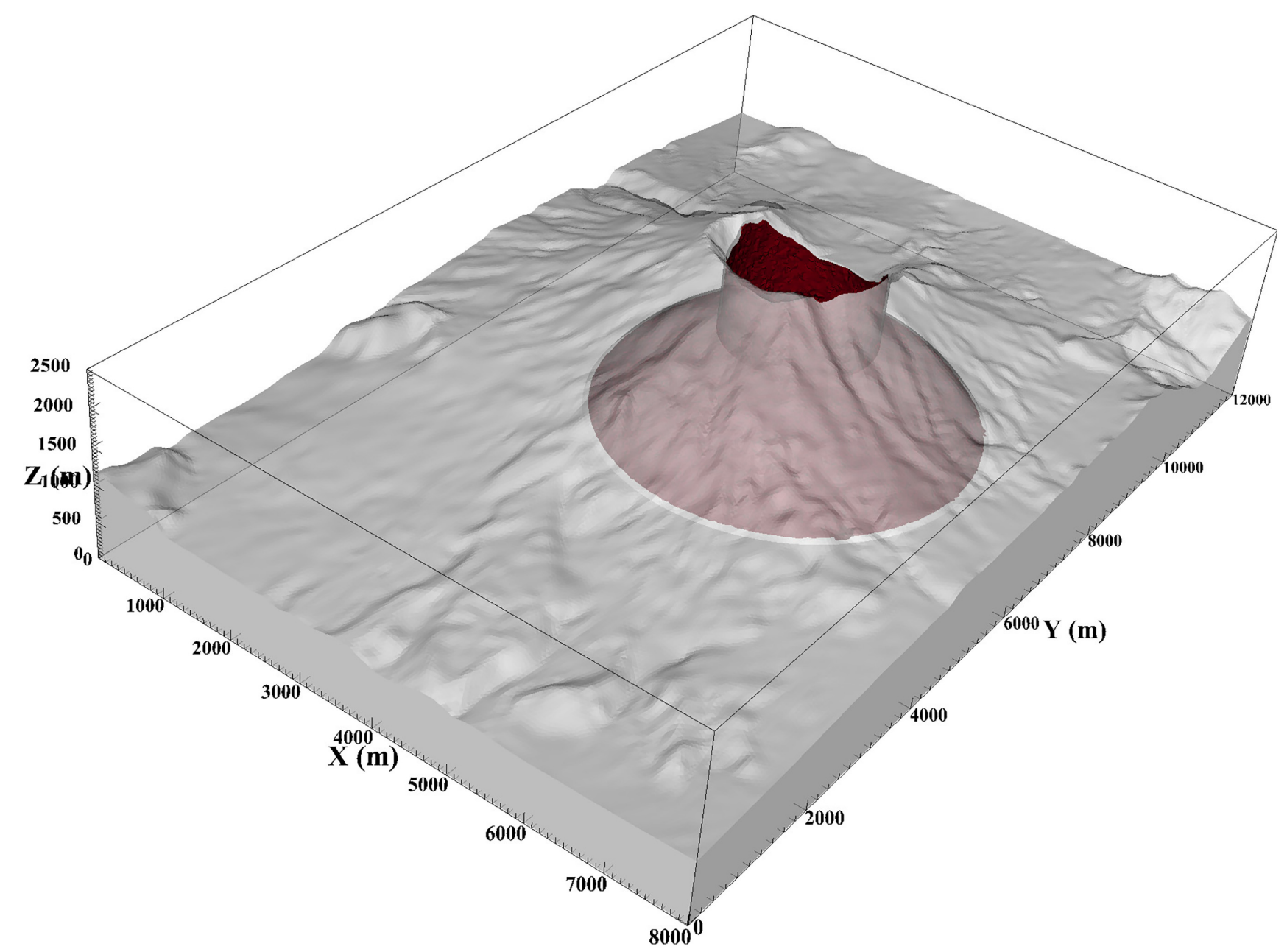

Figure 9. 3-D Model of the Mt. St Helens volcano covering an area of $12 \times 10 \mathrm{~km}^{2}$. The assumed magmatic chamber located at a depth of $1 \mathrm{~km}$ and the $500 \mathrm{~m}$ conduit are highlighted in red.

at a location contributes to a change of the observed electric field intensity $E_{s}$ at any of the stations. Regions with large sensitivity can be considered a focus of the inversion. Here we focus on the sensitivity for the electric field inversion. The approach can easily be translated for the chargeability inversion. As in most cases sensitivities for chargeability and conductivity show a similar distribution the discussion is focused on the conductivity case.

To define a sensitivity measure we consider the average of the logarithm of electric field intensity $E_{s}$ across all stations. The corresponding quantity $\Lambda_{s}$ is defined as

$\Lambda_{s}=-\int_{\Omega} \chi \log \left(\frac{E_{s}}{E_{s}^{\max }}\right) \mathrm{d} x$,

where $E_{s}$ is the electric field intensity for a given realization of the inversion target for experiment $s$. The non-negative coefficient $\chi$ is chosen as in the misfit function in eq. (17) to consider an average sensitivity over all stations. If the sensitivity for a particular station is of interest $\chi$ can be constructed with positive values at the neighbourhood of this station only. $E_{s}^{\max }$ is the maximum value of $E_{s}$ over the domain and is used a scaling fact to make the value of $\Lambda_{s}$ non-negative.

We monitor the change $\delta \Lambda_{s}$ of $\Lambda_{s}$ due to a change $\delta m_{1}$ of the given property function distribution $m_{1}$ :

$\delta \Lambda_{s}=\Lambda_{s}\left(m_{1}+\delta m_{1}\right)-\Lambda_{s}\left(m_{1}\right)$.
This change $\delta \Lambda_{s}$ is expressed in term of the change $\delta E_{s}$ to the electric field intensity as

$\delta \Lambda_{s}=\int_{\Omega} \chi \frac{\delta E_{s}}{E_{s}} \mathrm{~d} x=-\int_{\Omega} \frac{\chi}{E_{s}^{2}} \mathbf{E}_{s}^{t} \nabla \delta \phi_{s} \mathrm{~d} x$,

the increment $\delta \phi_{s}$ to the change to electric potential due property function increment $\delta m_{1}$. The quantity $\delta \Lambda_{s}$ denotes the (averaged) relative change of the electric field intensity at the stations. In a way similar to gradient calculation, the quantity $\delta \phi_{s}$ can be expressed in terms of $\delta m_{1}$. First an adjoint sensitivity $\Phi_{s}^{*}$ is calculated by solving the following equation

$\int_{\Omega} \sigma \nabla^{T} \psi \nabla \Phi_{s}^{*} d x=\int_{\Omega} \frac{\chi_{j}}{E_{s}} \mathbf{E}_{s}^{T} \nabla \psi d x$

for any smooth function $\psi$ (compare to eq. 27). With solution $\lambda_{s}$ of equation

$\int_{\Omega}\left(\lambda_{s} q+\alpha \nabla^{T} \lambda_{s} \nabla q\right) d x=\int_{\Omega} q \sigma^{\prime} \mathbf{E}_{s}^{T} \nabla \Phi_{s}^{*} d x$

for any smooth function $q$ (compare eq. 28) one obtains

$\delta \Lambda_{s}=\int_{\Omega} \lambda_{s} \delta m_{1} d x$ 


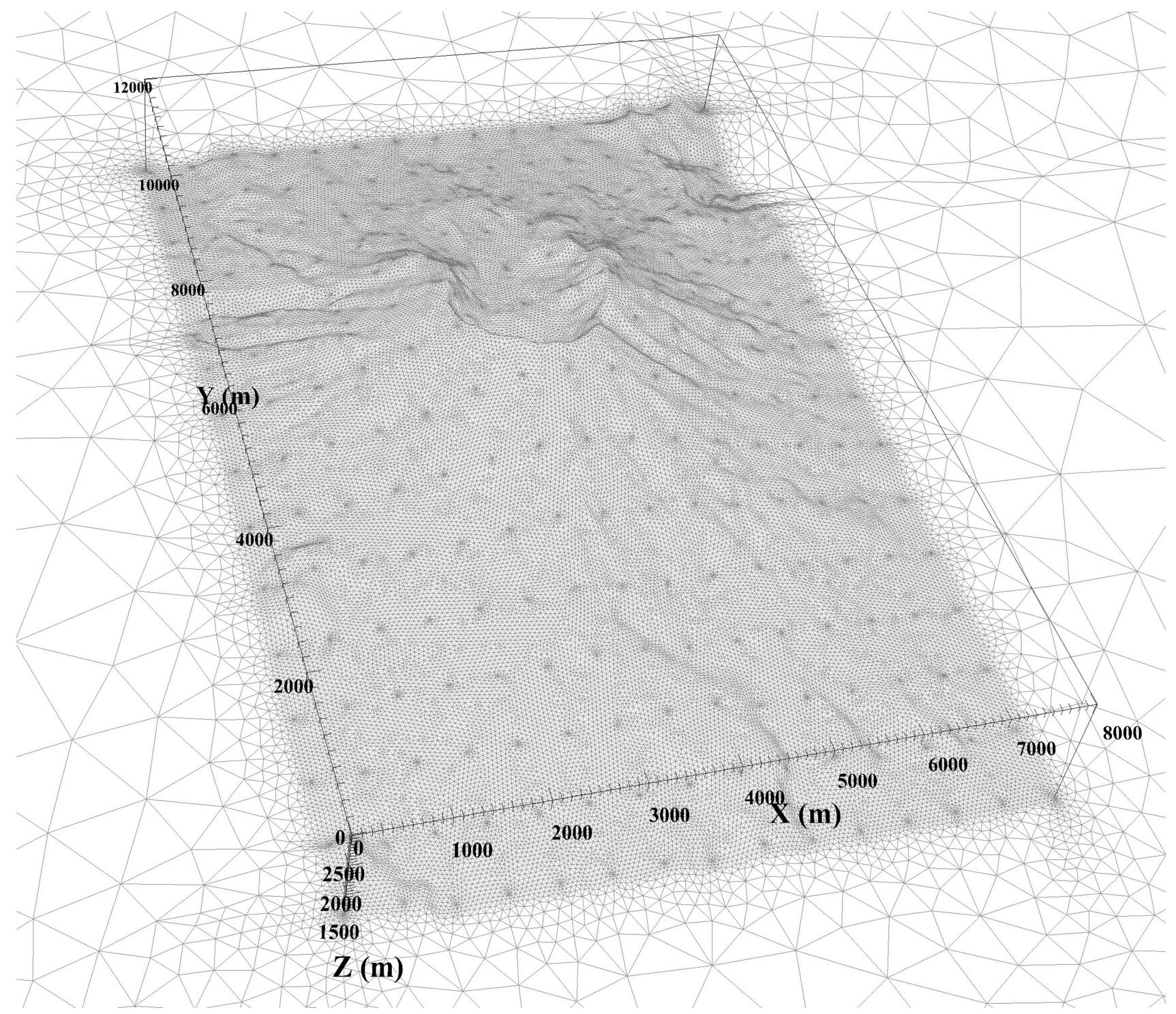

Figure 10. The finite element mesh of the volcano case study with 14.7 million tetrahedral elements. The core region is extending an area of $12 \times 10 \mathrm{~km}$ and is embedded into a larger outer region of size $40 \times 40 \times 15 \mathrm{~km}$ (not entirely shown). The mesh in the core regions is refined near the 256 position of the electrodes and stations while in the outer region a coarser grid is used. 199 observation stations are used. A total of 25 charging experiments are applied.

Choosing $\delta m_{1}$ as Dirac $\delta$-functions, we define the sensitivity $S_{s}(\mathbf{x})$ for any point $\mathbf{x}$ as

$S_{S}(\mathbf{x})=\left|\lambda_{S}(\mathbf{x})\right|$.

Backtracking the construction of $S_{s}(\mathbf{x})$ reveals that the sensitivity depends on the reference conductivity $\sigma_{0}$ and incorporates the impact of the smoothing length $\sqrt{\alpha}$. In fact for small values of $\alpha$ (or no smoothing at all) the sensitivity takes high values at the position of stations - a fact that can be deducted from the structure of the righthand side in eq. (37). For larger smoothing lengths, the sensitivity shows a more disseminated distribution away from the stations but the wider spread comes at the costs of reduction of the maximum sensitivity indicating a loss of resolution. In the examples presented later in this paper, the smoothing length is chosen as minimal distance of the stations in order to maintain a good sensitivity within the region covered by the survey. This choice has proven to deliver good results in other applications. However, it is emphasized that the smoothing length should be read as a geostatistical parameter (to be specific, the range of the spatial covariance) and needs to be chosen based on consideration of the geology or to be derived from observational data. Conversely, the spacing of station needs to be chosen to match the spatial covariance range when designing a survey in the field.

\section{BENCHMARK PROBLEMS}

We investigate now the robustness and limitations of the proposed approach using two different numerical tests both using unstructured 3-D finite element meshes. In both tests, the electric field intensity and induced polarization measurements are simulated. Synthetic data are collected using a set of stand-alone stations laid out on the ground surface while a number of current injections are performed in the region of interest as shown in Fig. 1. As for each current injection, the components of the electric field along the Cartesian coordinate axes are recorded at each station. A large data set of electric field measurements is obtained with a relatively small number of current injections. 


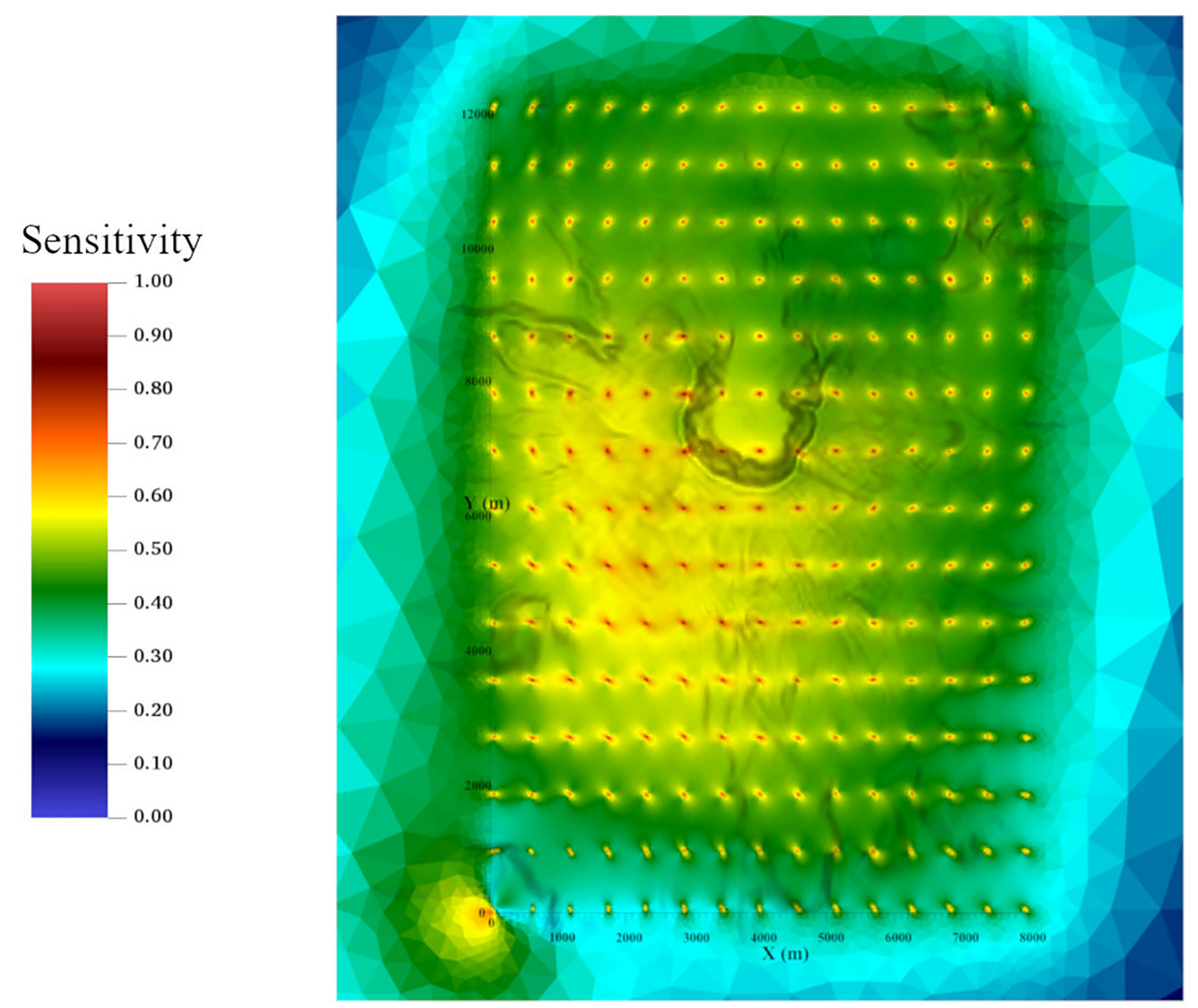

(a) Top view

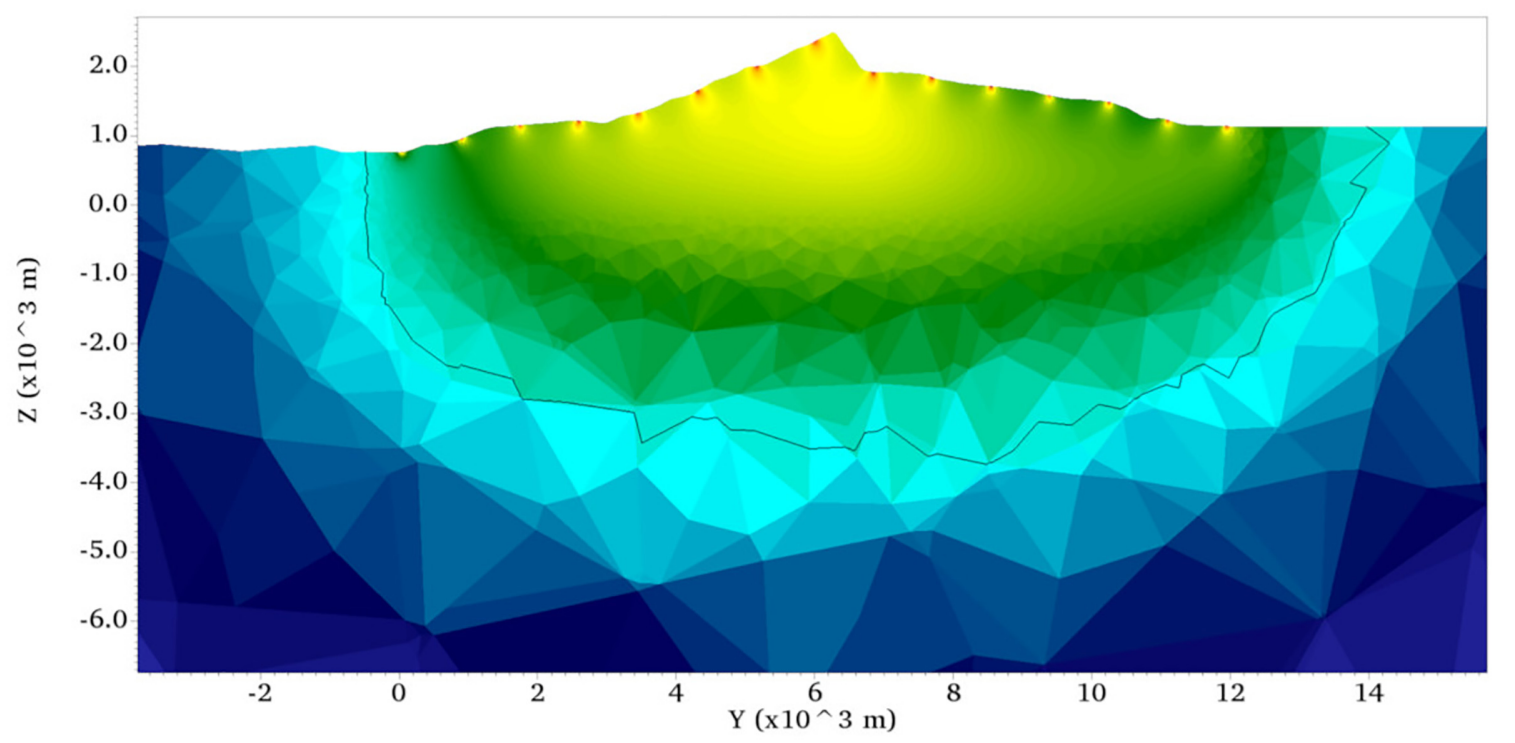

(b) Transect at $\mathrm{x}=4000 \mathrm{~m}$

Figure 11. Volcano: Sensitivity maps at top view (a) and transect $x=4000 \mathrm{~m}$ (b) for $\alpha=700^{2} \mathrm{~m}^{2}$. Regions of large sensitivity are colored in red. Blue regions are regions with low sensitivity. A sensitivity of 0.3 highlighted by the black contour line reaches down to depth of $4 \mathrm{~km}$ below the surface. The top view (a) reveals a strong bias towards the lower left corner which is the single charging electrode used by all experiments.

The first case study is a simple synthetic test and serves as a benchmark of the approach. For this case study, the medium is composed of a resistive and low chargeable background in which three cubic conductive and highly chargeable bodies are located. In the second tests, we take the digital elevation map (DEM) of the Mount St Helens volcano (Washington State, USA) to build a synthetic 3-D volcano with a complex topography. This synthetic volcano contains a vertical conduit connected to a deep magmatic chamber. 


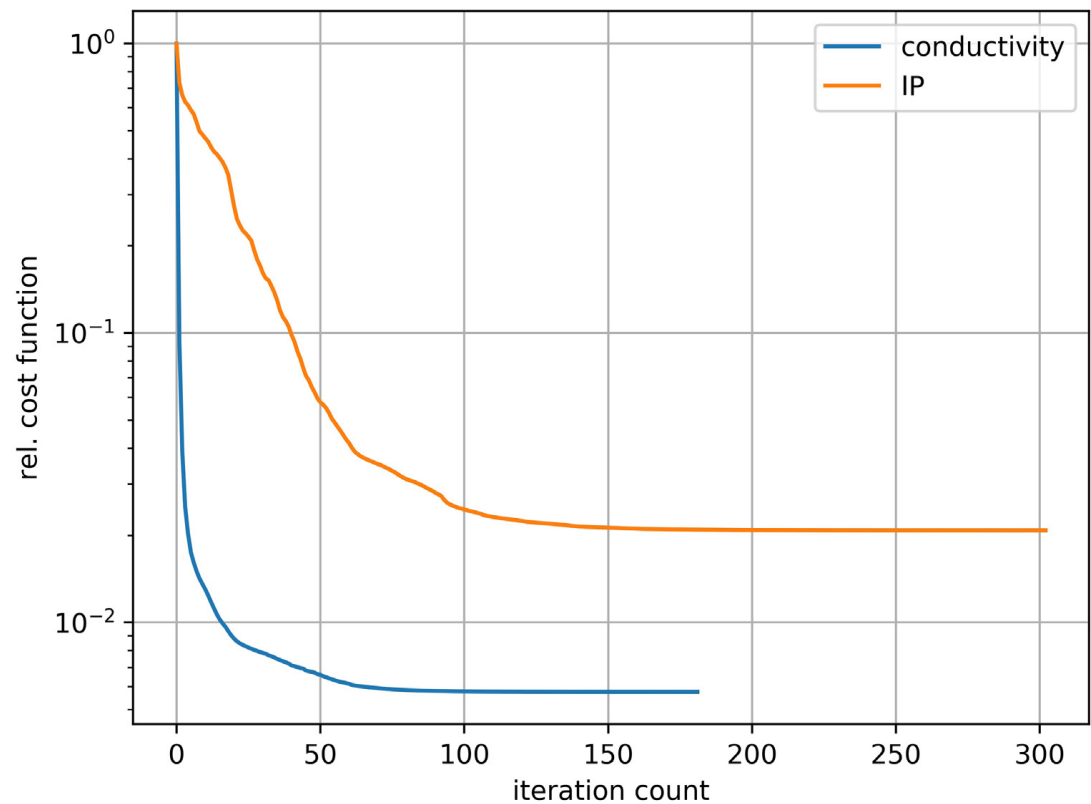

Figure 12. Volcano: Convergence history plot. Value of the cost function is shown relative its initial value. The iteration is terminated when the relative change of the unknown is lower than $10^{-4}$. For conductivity (blue curve), the cost function is quickly reduced initially but reduction starts to slow down around step 60. Convergence is slower for the IP chargeability (amber curve) and the reduction of the cost function is slowing down around the 120th step.

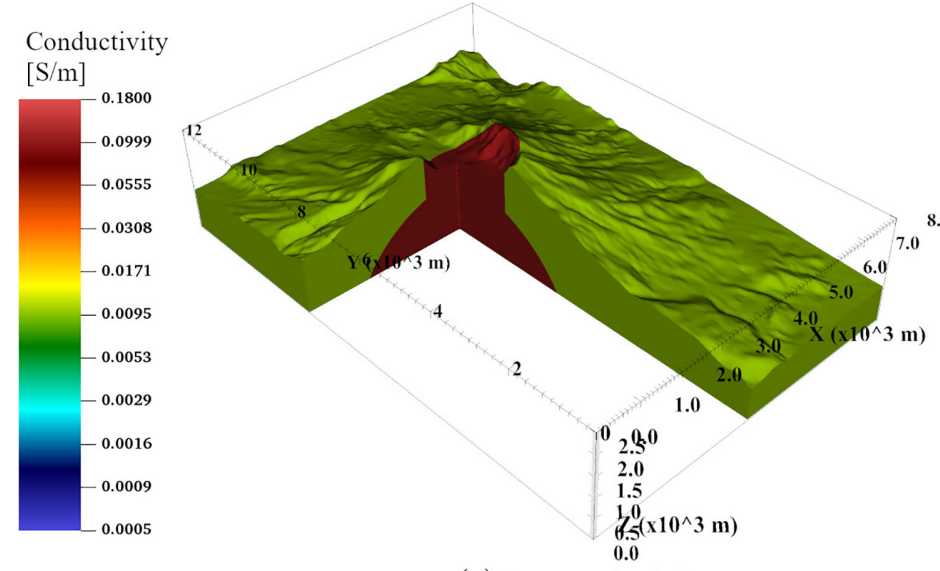

(a) True conductivity

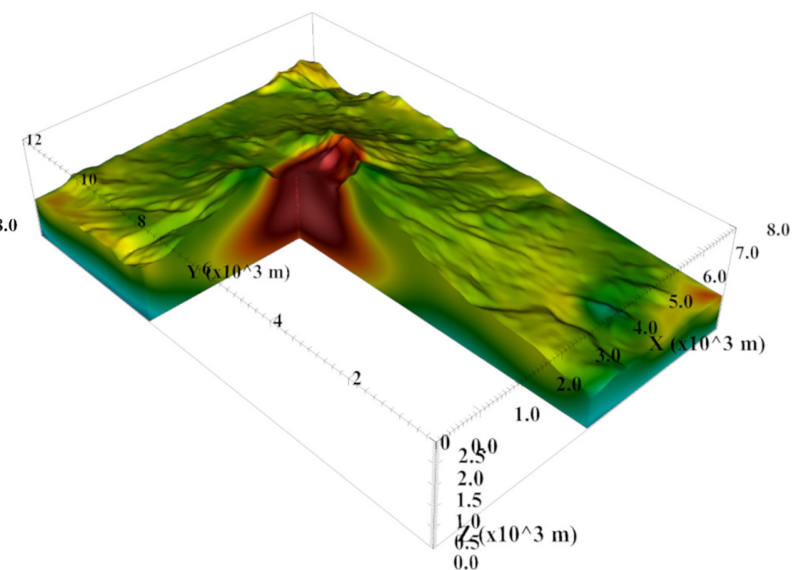

(b) Inverted conductivity

Figure 13. Volcano: 3-D view of the true conductivity distribution in (a) and inversion result (b). The spotted pattern of the conductivity near the surface is caused by the high-resolution topography. More details are shown in Fig. 16 for conductivity.

We have discretize the domain into a very fine mesh consisting of 8 million cells. The challenge here is twofold: (i) reconstructing the deep features of this large volcano and (ii) efficiently handling the computational burden associated to the use of such high-resolution mesh. The statistics of both case studies are reported in Tables 1 and 2 . We use the coefficient of determination $R^{2}$ over the region of interest to measure the quality of the recovered physical property $m_{i}$ versus the true distribution $m_{\text {true }, i}$.

\subsection{Case study 1: synthetic test case}

The domain of simulation of the first benchmark test covers a plain area of $4 \mathrm{~km} \times 4 \mathrm{~km}$ with a depth of $4 \mathrm{~km}$. It contains three localized anomalies in the shape of rectangular box of size $900 \times 900 \times$ $600 \mathrm{~m}^{3}$ at a depth $300 \mathrm{~m}$ as shown in Fig. 3(a). A total of 121 potential positions for charging electrodes and recording stations are arranged in a $11 \times 11$ grid with a spacing of $300 \mathrm{~m}$, see Fig. 3(b). Out of these 121 positions, 96 were randomly selected as positions for recording station while the remaining 25 were kept as potential positions for charging electrodes. Four additional charging electrodes were introduced at the centre of each face of the grid located with on offset of $300 \mathrm{~m}$ outside the grid, see Fig. 3(b). For each of these four additional electrodes 10 charging electrodes were randomly chosen to perform charging experiments. This results in 40 charging experiments with a total of 3840 recordings. The reason behind switching locations of the injecting electrodes during for each current injection is to stimulate different areas of the subsurface and though maximizing the sensitivity and the information that we obtain on the structure of the subsurface. The number of charging experiments had been limited to 40 in order to consider potential budget limitations as they might be set in a field application. As each charging experiment requires the solution of a forward 


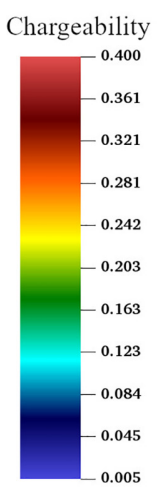

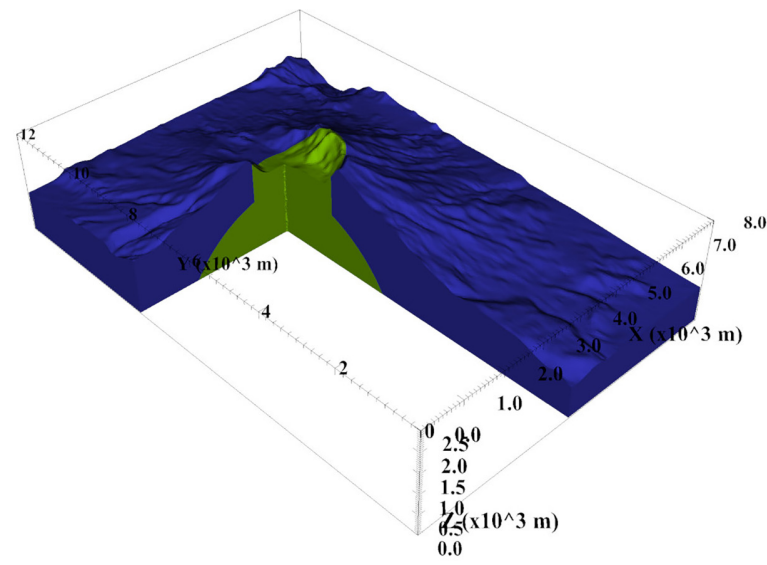

(a) True Chargeability

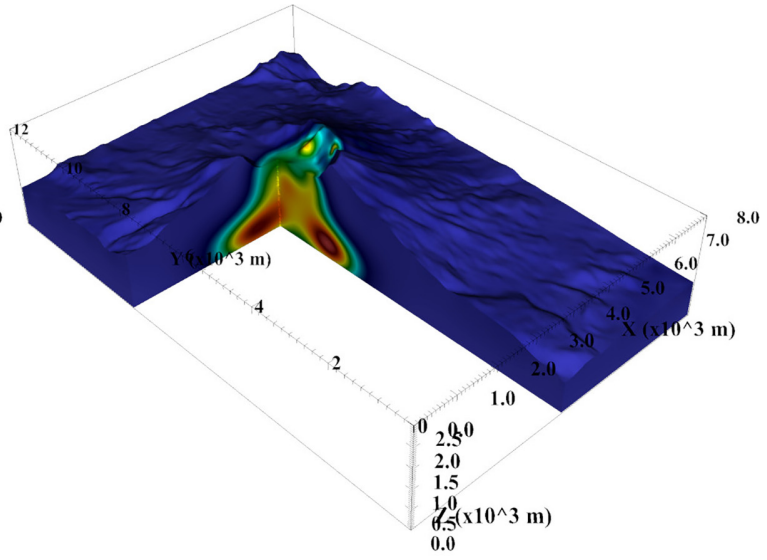

(b) Inverted Chargeability

Figure 14. Volcano: 3-D view of the true chargeability (a) and the inversion result in (b). Again, the spotted pattern of the chargeability near the surface can be attributed to topography.
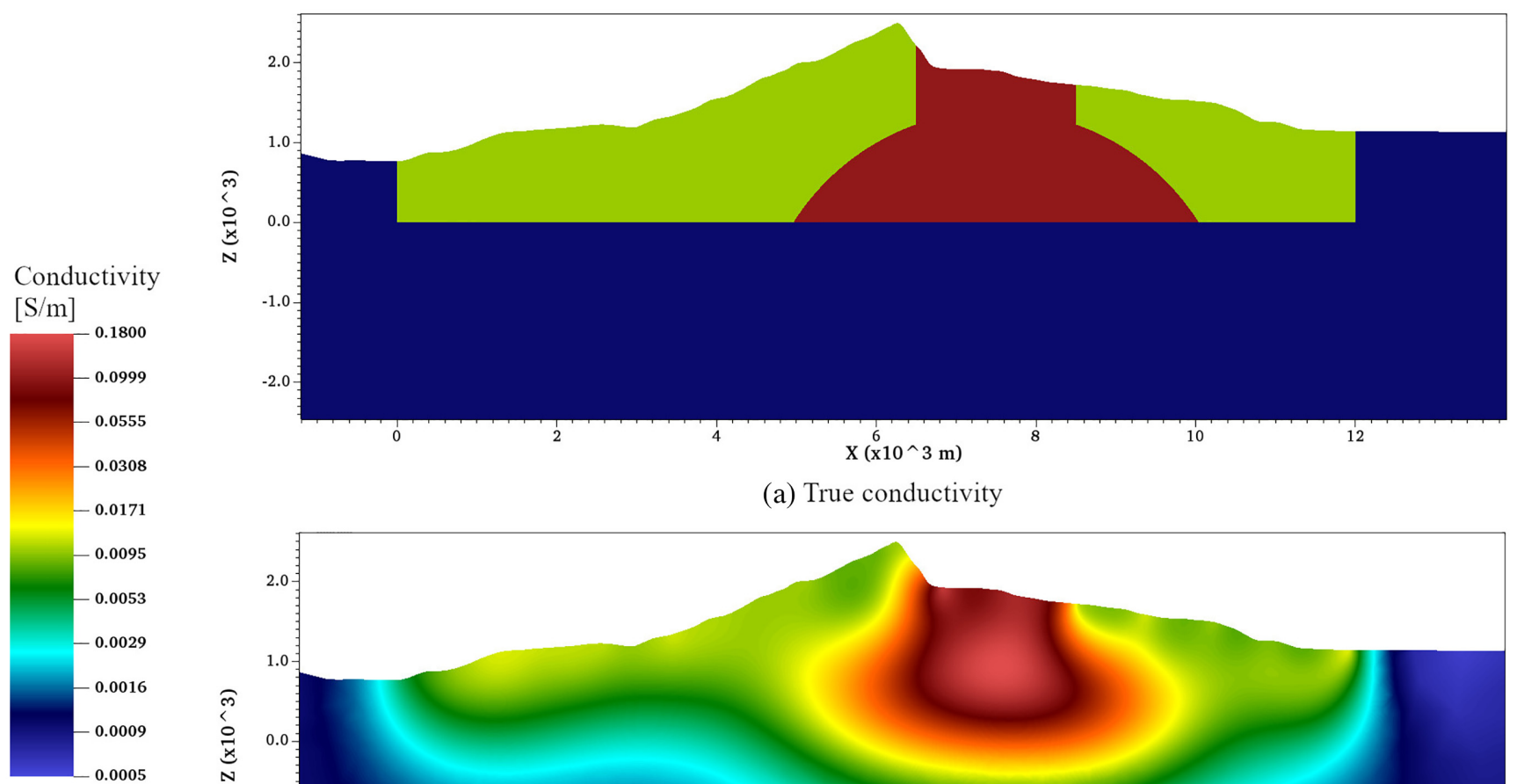

(a) True conductivity

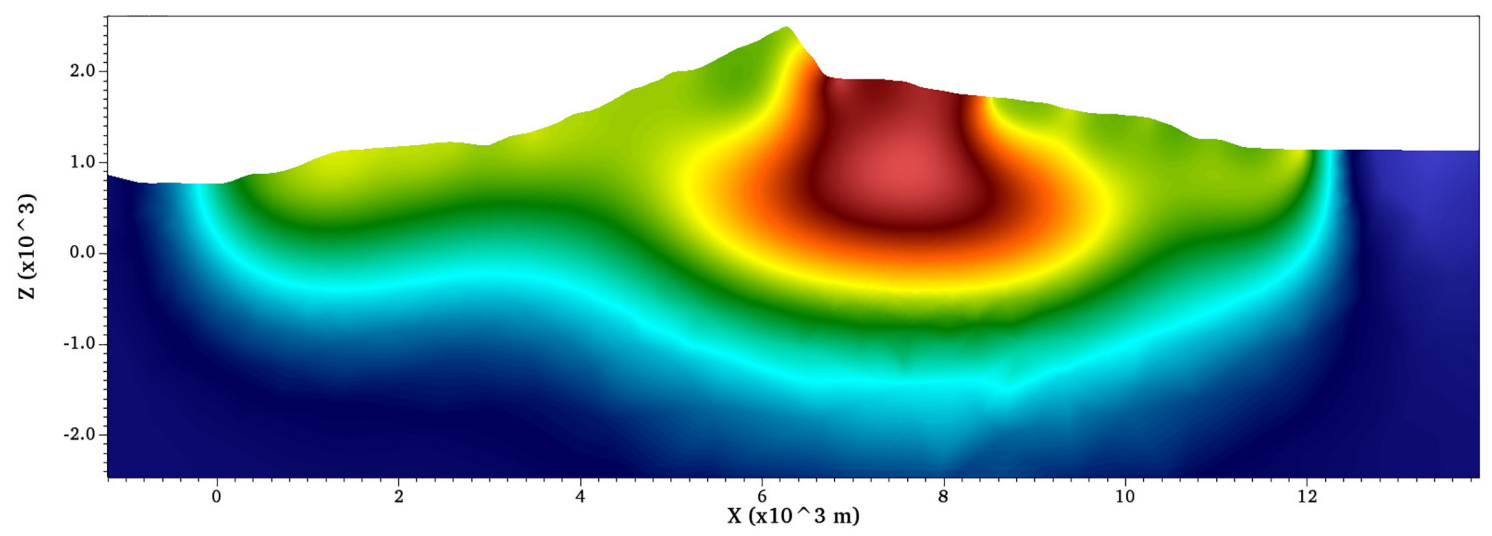

(b) Inverted conductivity

Figure 15. Volcano: True (a) and inverted conductivity $\sigma(\mathrm{b})$ at $x=4 \mathrm{~km}$. Due to the effect of the regularization the interface of the conduit and chamber with a relative high conductivity is smeared and the peak conductivity in the inversion results overpredicts the true conductivity $\left(0.18 \mathrm{Sm}^{-1}\right.$ versus $\left.0.10 \mathrm{Sm}^{-1}\right)$. Similarly, the interface between the volcano and the surrounding region is smooth.

problem this restriction also reduces the cost for the inversion. It is pointed out that for the presented approach computational costs are independent from the number of observation stations.

The domain of simulation is discretized with unstructured mesh using 748,350 tetrahedron elements, see Fig. 4. The mesh has been generated using the gmsh package (Geuzaine \& Remacle (2009)). The true conductivity model is set as follows: $0.001 \mathrm{~S} \mathrm{~m}^{-1}$ for the background and $0.01 \mathrm{~S} \mathrm{~m}^{-1}$ for the anomaly, while the chargeability values are 0.01 for the background and 0.20 for the anomalies, see Fig. 3 for details. Following eq. (4), we use the norm of the electric field as input data for reconstructing the conductivity field and the apparent chargeability described in eq. (10) for constructing the chargeability field. The data are contaminated with a 5 per cent Gaussian noise. 


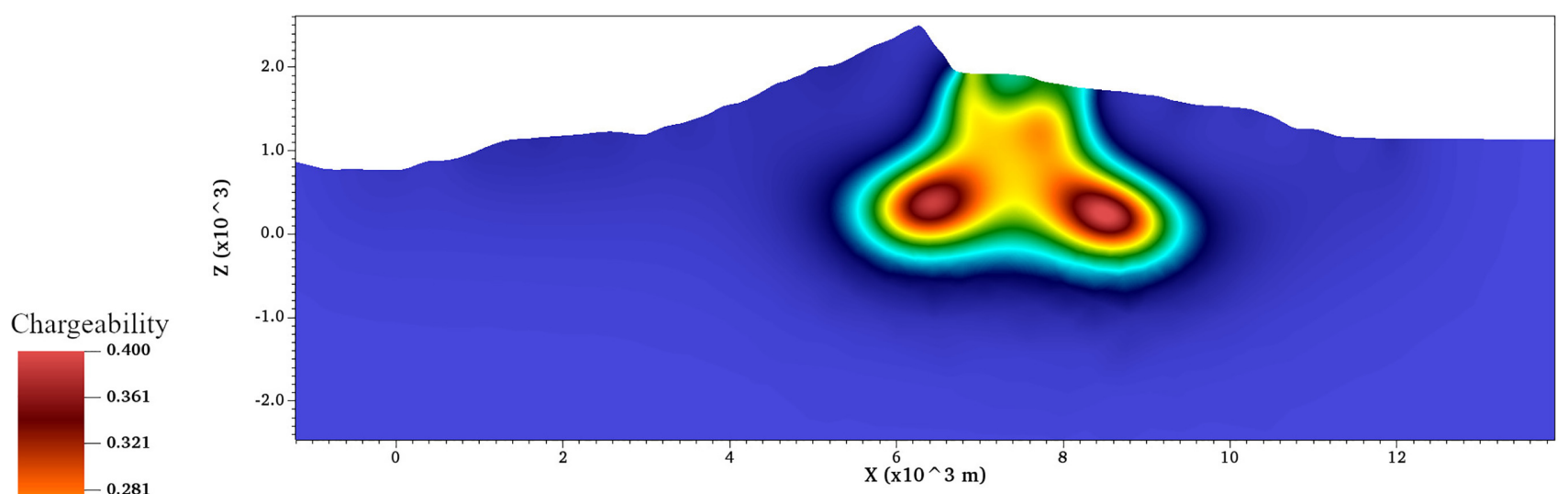

(a) True chargeability

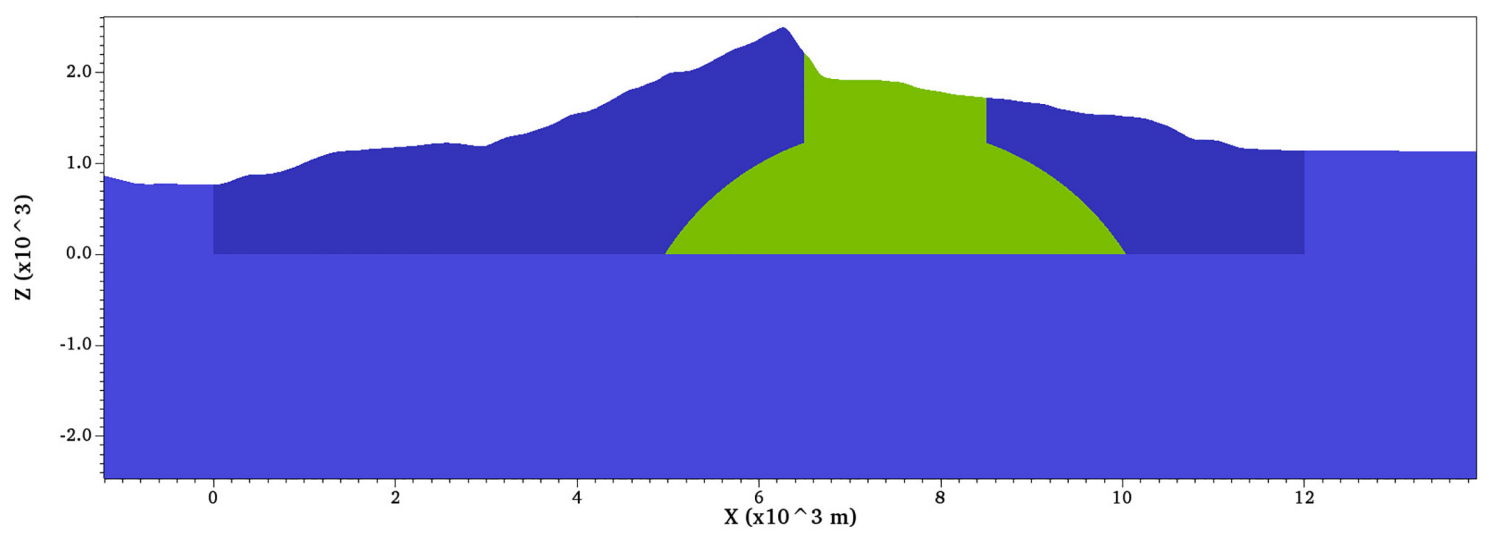

(b) Inverted chargeability

Figure 16. Volcano: True (a) and inverted modified chargeability $\gamma$ (b) at $x=4 \mathrm{~km}$. The shape of the chamber and conduit is well reproduced with a shaper representation of the interfaces to the volcano and the basement. Again, due to the effect of the regularization the chargeability is predicted with a higher value than in the true model $(0.4$ versus 0.2$)$. The lower chargeability of 0.02 in the volcano has not been reproduced in the inversion and the interface to the basement with an even lower chargeability is not apparent.

Fig. 5 presents the distribution of sensitivity for various values for the length scale parameter $\alpha$ (with $\sigma_{0}=0.001 \mathrm{~S} \mathrm{~m}^{-1}$ and $m_{1}=0$ ). The sensitivity is averaged over all 40 charging experiments. As discussed earlier, the sensitivity is highest near the recording stations but is spread out further vertically as well as horizontally for larger values of $\alpha$. For the inversion we set the smoothing length scale to the distance of the stations $\left(\alpha=300^{2} \mathrm{~m}^{2}\right)$ which achieves a good sensitivity larger than 0.3 below $1 \mathrm{~km}$ depth defining the bottom of the anomalies. The regularization parameters is set to $\mu_{i}=2 \times 10^{-15}$. As that the smallest value for which the regularization contributions $\mu_{i} R\left(m_{i}\right)$ to the corresponding cost functions become about one order of magnitude lower than the respective misfits $M_{i}\left(p_{i}\right)$. These values have been obtained through various trial runs. After 424 iterations for the conductivity inversion and 302 iteration for the chargeability inversion, the BFGS iteration is terminated due to small changes in the property function (relative change smaller than $10^{-4}$ ). Fig. 6 plots the reduction of the cost function over the iteration in the convergence history. Due to the line search applied in each iteration step, the cost function monotonously decreases. The history shows the typical initially steep decrease of the misfit function. Then it stabilizes as the iteration is progressing. The steep drop at step count 60 can be attributed to the fact that in the used implementation the BFGS iteration is restarted after every 60 steps in order to reset the approximation of the inverse of the Hessian built using a sliding window of 25 gradients. The cost functions for the conductivity and chargeability both are monotonously reduced with the iteration count while the iteration is terminated when the relative change in the unknown is below the tolerance $10^{-4}$. The results are shown in Fig. 7 for the conductivity and Fig. 8 for the modified chargeability. One can see that in both cases, the anomalies are wellreconstructed both in terms of their locations and their magnitudes. In the results for conductivity in Fig. 7d) the method is also able to well separate the neighbouring anomalies. The smoothness effect is inherent to the regularization approach. As highlighted in the error plots Figs 7(e) and (f) and Figs 8(e) and (f) the property values at anomalies are slightly overpredicted while the interfaces to the background are smoothed still producing a physically meaningful inversion result. The coefficients of determination $R^{2}$ for the predicted versus the true properties is slightly better for the inversion of conductivity, see Table 1.

\subsection{Case study 2: synthetic volcano}

This case study aims at checking the robustness of the proposed approach under conditions that mimic those of real field application. The simulation domain reproduces the topography of the Mount St Helens volcano (Washington State, USA). We took a $1 \mathrm{~m}$ resolution Digital Elevation Map (DEM) model of this volcano that is freely 
accessible from the website https://nationalmap.gov/elevation.html and then assembled the 3-D geometrical model of the volcano. The resulting model covers an area of $12 \mathrm{~km} \times 10 \mathrm{~km}$, has a complex geometry with it summits at $1230 \mathrm{~m}$ (see Fig. 9). The synthetic volcano has an internal structure composed of a $30 \mathrm{~m}^{3}$ magmatic chamber located at a depth of $1 \mathrm{~km}$ and connected from its top to a $500 \mathrm{~m}$ conduit that vertically expand to the summit of the volcano. The survey uses 224 stations with 25 current injections leading to 551 measurements.

The high resolution of the DEM model combined with the complexity of the topography makes it necessary to finely discretize the simulation domain to capture the details of the geometry. Therefore, we have used a high-resolution mesh that contains 14.7 million tetrahedral elements (see Fig. 10). We used the COMSOL package to generate the mesh (COMSOL 2020). For this study the COMSOL mesh generator was preferred over the gmsh package as it provides better capabilities to mesh a domain with topography. The true conductivity and chargeability fields are designed in such a way to make both the conduit and the magmatic chamber more electrically conductive and chargeable due to the strong alteration halo around these features. We assign a conductivity of $0.1 \mathrm{~S} \mathrm{~m}^{-1}$ to magmatic chamber and the conduit and $0.01 \mathrm{~S} \mathrm{~m}^{-1}$ to the remaining part of the volcano while a value of $0.001 \mathrm{~S} \mathrm{~m}^{-1}$ is used as background conductivity outside the volcano. These values will be discussed in greater details in the next papers of the present series. A chargeability of 0.2 is assigned to the magmatic chamber and the conduit while a value 0.02 is used as the chargeability in remaining part of the volcano. The background chargeability is set to 0.005 . The synthetic survey data a perturbed with 5 per cent Gaussian noise.

For the inversion, we choose the length scale parameter $\alpha=$ $500^{2} \mathrm{~m}^{2}$ where the $500 \mathrm{~m}$ is the distance of the measuring stations and regularization parameters $\mu_{i}=5 \times 10^{-15}$. Again, this value for $\mu_{i}$ has been obtained through test runs and picking the smallest value for which the contributions from the regularization term to the cost function become about one order of magnitude smaller than the misfit. The survey is biased towards one corner of the survey array as in contrast to the synthetic case dipoles sharing a single electrode are used for charging. To illustrate this point, Fig. 11 displays the sensitivity distribution for a slightly larger length scale parameter $\alpha=700^{2}$ for which the effect is more exposed. By construction, the iteration reduces the cost function monotonously (see Fig. 12). The stopping criterion is reached after 180 iterations for the conductivity and 301 iterations for the chargeability (see Table 2). Similarly, to the synthetic case but more clearly here, we see that the convergence for conductivity inversion is faster in comparison to the chargeability inversion. This can be attributed to the fact that as a relative difference the evaluation of chargeability is emphasizing variations in the electric field intensity in the misfit leading to more ill-posed inversion problem. The misfit is reduced by over three order magnitudes for the conductivity but only two order of magnitudes for the chargeability, see Table 2.

Figs 13 and 14 give a 3-D overview of the inversion results with more details shown in Figs 15 and 16. Despite the low number of current injections (25) the structure of the volcano and more interestingly its deep features down to $3 \mathrm{~km}$ below the summit are fairly-well reconstructed. Due to the regularization interfaces are smoothed while the peak value for the high conductivity and chargeability against the resistant basement with negligible chargeability are over-predicted by the inversion. In addition the coefficients of determination $R^{2}$ for the predicted versus the true properties is better for the inversion of conductivity (see Table 2). Besides the larger number of steps to completion, these last observations can also be attributed to the roughening effect of the electric field difference in the chargeability calculation in eq. (7).

The inversion method presented here is tailored to solve largescale inversions as demonstrated in the synthetic volcano which has a total of over 130 million unknowns including the solutions of the forward and adjoint problems modeling the 25 charging experiments. The inversion for conductivity run for just under $6 \mathrm{hr}$ using 200 cores on a compute cluster with dual socket compute nodes with 126 GB per node and Intel Xeon CPU E5-2660 processors at $2.60 \mathrm{GHz}$ with 10 cores each (no hyperthreading) Interconnect was a 4 XDDR Infiniband. We have been able solve such a large inversion problem using a highly parallelized code as in contrast to conventional methods the approach presented in this paper does not require to assemble and invert a dense matrix. Moreover it makes use of an explicit representation of the cost function Jacobian with respect to the inversion unknown, which can easily be derived in a continuous formulation of the inversion problem with a downstream FEM approximation, see Codd \& Gross (2017) for a more detailed discussion.

\section{CONCLUSION}

We have developed an innovative approach for inverting the primary and secondary electric field components in an induced polarization field experiment in order to image the structure of the subsurface in terms of electrical conductivity and chargeability fields. Using the two components of the electrical fields remove issues associated with $2 \mathrm{D}$ profiles and related to the distorsion of the electrical field generating spurious negative apparent resistivity and chargeability values. The method can be easily adapted to image the relaxation times as well. With the recently developed new induced polarization equipment, one can directly measure the components of the electric fields on stand-alone stations that do not need to be connected through cables. This improves the quality of the data by reducing the capacitive coupling effects and makes it possible to carry out large-scale true 3-D IP surveys on rough topographic areas such as volcanoes. The current use of photogrammetry with drones can be used to get quickly (in few hours) the required 3-D tomography. The proposed inversion scheme takes the electric field intensity as coordinate system invariant input data and returns output the electrical conductivity and chargeability distributions. The new method presents several advantages, including: (i) it is suitable for large-scale applications as in contrast to conventional approached it does not require an explicit assembly of the Jacobian matrices; (ii) through the support of unstructured meshes it provide the possibility to easily perform inversions on complex geometries; (iii) it eliminates the local geometrical configuration of stations through the use of orientation invariant quantities of electric field data and (iv) it comes with a 3-D sensitivity maps helping in survey design prior to optimize the location of injecting electrodes and recording stations configuration to obtain the best imagery results.

We have successfully validated this new approach on a large-scale synthetic IP survey that mimic true field conditions as they can for instance be encountered in the field targeting a 3-D mapping of a volcano. The presented application demonstrates the great potential of electric field surveys in volcanology especially when targeting an image of the volcanoes' deep internal structures for instance to improve the understanding of their hydrothermal systems or to monitor deep volcanic activities and their plumbing systems. Armed with this proof of concept, future works are geared towards the collection and inversion of IP data for volcanoes. 


\section{ACKNOWLEDGEMENTS}

We thank the Editor, an anonymous referee and Andrew Binley for their constructive comments. We thank IRIS for fruitful discussions regarding the use of the two components of the electrical field in conductiivty and induced polarization survey, especially Catherine Truffert and Julien Gance, who have initially stimulated our interest for this subject. This work was enabled by AuScope and the Australian Government via the National Collaborative Research Infrastructure Strategy (NCRIS): auscope.org.au. Code Accessibility: The python implementation of the presented inversion method including the scripts and data to run the two examples are available under the Apache License 2.0 at https://github.com/LutzGross/fingal [visited Jan 2021]. The implementation is based on the esys-escript module, see https:/github.com/esys-escript/esys-escript.github.io [visited January, 2021]. We used version 5.6 available at doi.org/10.1 4264/uq1.2020.966. The data used for the simulations are available upon request to the authors of the paper.

\section{REFERENCES}

Brandt, A., McCoruick, S. \& Huge, J., 1985. Algebraic multigrid (AMG) for sparse matrix equations, in Sparsity and its Applications, Cambridge Univ. Press.

Brandt, A., 1986. Algebraic multigrid theory: the symmetric case. Appl. Math. Comput., 19, 23-56.

Codd, A.L. \& Gross, L., 2017. Electrical resistivity tomography using a finite element based BFGS algorithm with algebraic multigrid preconditioning. Geophys. J. Int., 212, 2073-2087.

COMSOL, 2020. COMSOL Multiphysics $@$ v. 5.4. www.comsol.com. COMSOL AB, Stockholm, Sweden [visited Oct 2020].

Fiandaca, G., Ramm, J., Binley, A., Gazoty, A., Christiansen, A.V. \& Auken, E., 2012. Resolving spectral information from time domain induced polarization data through 2-D inversion. Geophys. J. Int., 192, 631-646.

Fikos, I. et al., 2012. Electrical resistivity tomography study of Taal volcano hydrothermal system, Philippines. Bull. Volcanol., 74, 1821-1831.

Ghorbani, A., Revil, A., Coperey, A., Ahmed, A.S., Roque, S., Heap, M., Grandis, H. \& Viveiros, F., 2018. Complex conductivity of volcanic rocks and the geophysical mapping of alteration in volcanoes. J. Volc. Geotherm. Res., 357, 106-127.

Gross, L., Altinay, C. \& Shaw, S., 2015. Inversion of potential field data using the finite element method on parallel computers. Comput. Geosci., 84, 61-71.

Geuzaine, C. \& Remacle, J.-F., 2009. Gmsh: a three-dimensional finite element mesh generator with built-in pre- and post-processing facilities. Int. J. Numer. Methods Eng., 79, 1309-1331.

Gurin, G., Tarasov, A., Ilyin, Y. \& Titov, K., 2013. Time domain spectral induced polarization of disseminated electronic conductors: laboratory data analysis through the Debye decomposition approach. J. Appl. Geophys., 98, 44-53.

Günther, T., Rücker, C. \& Spitzer, K., 2006. Three-dimensional modelling and inversion of DC resistivity data incorporating topography-II. Inversion. Geophys. J. Int., 166(2), 506-517.

Johnson, T.C. \& Thomle, J., 2017. 3-D decoupled inversion of complex conductivity data in the real number domain. Geophys. J. Int., 212, 284296.

Johnson, T.C., Versteeg, R.J., Ward, A., Day-Lewis, F.D. \& Revil, A., 2010. Improved hydrogeophysical characterization and monitoring through parallel modeling and inversion of time-domain resistivity and inducedpolarization data. Geophysics, 75(4), WA27-WA41.

Lesparre, N., Grychtol, B., Gibert, D., Komorowski, J.-C. \& Adler, A., 2014. Cross-section electrical resistance tomography of La Soufrière of Guadeloupe lava dome. Geophys. J. Int., 197, 1516-1526.

Lewicki, J., Connor, C., St-Amand, K., Stix, J. \& Spinner, W., 2003. Selfpotential, soil $\mathrm{CO}_{2}$ flux, and temperature on Masaya volcano, Nicaragua, Geophys. Res. Lett., 30,.
Li, Y. \& Oldenburg, D.W., 2000. 3-D inversion of induced polarization data. Geophysics, 65, 1931-1945.

Loke, M.H., 2003. RES2DINV rapid 2-D resistivity and IP inversion using the least squares method, Manual. Geotomo Software, Penang.

Karaoulis, M., Revil, A., Werkema, D.D., Minsley, B., Woodruff, W.F. \& Kemna, A., 2011. Time-lapse 3D inversion of complex conductivity data using an active time constrained (ATC) approach. Geophys. J. Int., 187, 237-251.

Karaoulis, M., Revil, A., Werkema, D.D., Tsourlos, P. \& Minsley, B.J., 2013. IP4DI: a software for time-lapse 2D/3D DC-resistivity and induced polarization tomography, Comput. Geosci., 54, 164-170.

Mondal, S., Olsen-Kettle, L.M. \& Gross, L., 2020. Regularization of continuum damage mechanics models for 3-D brittle materials using implicit gradient enhancement, Comput. Geotech., 122, doi:10.1016/j.compgeo.2020.103505.

Ogawa, Y. et al., 1999. Wide-band magnetotelluric measurements across the Taupo Volcanic Zone, New Zealand-preliminary results, Geophys. Res. Lett., 26(24), 3673-3676.

Oldenburg, D.W. \& Li, Y., 1994. Inversion of induced polarization data. Geophysics, 59, 1327-1341.

Pidlisecky, A., Haber, E. \& Knight, R., 2007. RESINVM3D: a 3D resistivity inversion package. Geophysics, 72(2), H1-H10.

Prokopenko, A., Hu, J.J., Wiesner, T.A., Siefert, C.M. \& Tuminaro, R.S., 2014. MueLu user's guide 1.0. Techical Report SAND2014-18874, Sandia National Laboratories.

Revil, A., Saracco, G. \& Labazuy, P., 2003. The volcano-electric effect. J. geophys. Res., 108, doi:10.1029/2002JB001835.

Revil, A., Gailler, L.-S., Gresse, M., Cluzel, N., Panwar, N. \& Sharma, R., 2018. Electrical conductivity and induced polarization investigations at Kilauea volcano, Hawai'i. J. Volc. Geotherm. Res., 368, 31-50.

Revil, A., Johnson, T. \& Finizola, A., 2010. Three-dimensional resistivity tomography of Vulcan's forge, Vulcano Island, southern Italy. Geophys. Res. Lett., 37, doi:10.1029/2010GL043983.

Revil, A., Murugesu, M., Prasad, M. \& Le Breton, M., 2017. Alteration of volcanic rocks: A new non-intrusive indicator based on induced polarization measurements. J. Volc. Geotherm. Res., 341, 351-362.

Revil, A., Breton, M.Le, Niu, Q., Wallin, E., Haskins, E. \& Thomas, D.M., 2017 a. Induced polarization of volcanic rocks. 1. Surface versus quadrature conductivity, Geophys. J. Int., 208, 826-844.

Revil, A., Breton, M.Le, Niu, Q., Wallin, E., Haskins, E. \& Thomas, D.M., $2017 \mathrm{~b}$. Induced polarization of volcanic rocks. 2. Influence of pore size and permeability, Geophys. J. Int., 208, 814-825.

Revil, A., Qi, Y., Ghorbani, A., Coperey, A., Soueid Ahmed, A., Finizola, A. \& Ricci, T., 2019. Induced polarization of volcanic rocks. 3. Imaging clay cap properties in geothermal fields, Geophys. J. Int., 218(2), 1398-1427.

Rücker, C., Günther, T. \& Wagner, F.M., 2017. pyGIMLi: An open-source library for modelling and inversion in geophysics. Comput. Geosci., 109, $106-123$.

Schaa, R., Gross, L. \& Du Plessis, J., 2016. PDE-based geophysical modelling using finite elements: examples from $3 \mathrm{D}$ resistivity and $2 \mathrm{D}$ magnetotellurics. J. Geophys. Eng., 13, S59-S73.

Schlumberger, C., 1920, Etude sur la prospection électrique du soussol, Gauthier-Villars, Paris. The second edition without modification is available at https://gallica.bnf.fr/ark:/12148/bpt6k64569898.texteImage. English version, translated by Sherwin F. Kelly: "Study of underground electrical prospection". Available for instance at here: https://archive.org/details/studyofundergrou00schlrich/page/n37.

Soueid Ahmed, A. et al., 2018. 3D electrical conductivity tomography of volcanoes. J. Volc. Geotherm. Res., 356, 243-263.

Truffert, C., Leite, O., Gance, J., Texier, B. \& Bernard, J., 2017. Large 3D resistivity and induced polarization acquisition using the Fullwaver system: towards an adapted processing methodology. EGU General Assembly Conference Abstracts, 19, 12775.

Zhou, J., Revil, A., Karaoulis, M., Hale, D., Doetsch, J. \& Cuttler, S., 2014. Image-guided inversion of electrical resistivity data, Geophys. J. Int., 197, 292-309.

Zienkiewicz, O.C., Taylor, R.L. \& Zhu, J.Z., 2005. The Finite Element Method: Its Basis and Fundamentals, Elsevier. 